\title{
A comparison of various noncommuting conditions in metric fixed point theory and their applications
}

\author{
Ravi P Agarwal ${ }^{1,2}$, Ravindra K Bisht ${ }^{3}$ and Naseer Shahzad ${ }^{2 *}$ \\ In memory of Professors SP Singh and MA Al-Thagafi.
}

\author{
"Correspondence: \\ nshahzad@kau.edu.sa \\ ${ }^{2}$ Department of Mathematics, King \\ Abdulaziz University, P.O. Box 80203 \\ Jeddah, 21589, Saudi Arabia \\ Full list of author information is \\ available at the end of the article
}

\begin{abstract}
This paper presents a survey that aims to provide a brief historical account of the development through the definitions and comparison of weaker forms of commuting mappings set brought together from some applications oriented point of view. MSC: $47 \mathrm{H} 10 ; 54 \mathrm{H} 25 ; 41 \mathrm{~A} 50$

Keywords: common fixed point; $R$-weakly commuting mappings; compatible mappings; weakly compatible mappings; starshaped set; best approximation; best simultaneous approximation; convex metric space; nonexpansive mapping; $R$-subcommuting; $R$-subweakly commuting mappings
\end{abstract}

\section{Introduction and preliminaries}

In 1957, Isbel [1] (see also [2]) posed an interesting problem: If $\left\{f_{n}\right\}_{n \in \mathbb{N}}$ is a family of commuting continuous self-mappings of $[0,1]$ then do there exist common fixed points for $\left\{f_{n}\right\}$ ? It was only in 1969 that Boyce [3] and Huneke [4] independently proved that there exist two continuous commuting self-mappings of the unit interval $[0,1]$ without a common fixed point.

The well-known Banach contraction principle states that if a self-mapping $f$ of a complete metric space $(X, d)$ satisfies the condition

(i) $d(f x, f y) \leq k d(x, y), 0 \leq k<1$,

for each $x, y \in X$, then $f$ has a unique fixed point, that is, there exists a unique $z \in X$ such that $f(z)=z$.

A fixed point of a self-mapping of a metric space $X$ can also be considered to be a common fixed point of $f$ with the identity mapping on $X$. An innate question that comes up in this context is whether the identity mapping can be replaced by another self-mapping $g$ of $X$ to obtain common fixed points of $f$ and $g$. In 1968, Goebel [5] studied this problem and obtained the following coincidence theorem.

Theorem 1.1 Let $A$ be an arbitrary set and $X$ be a metric space with the metric d. Suppose, moreover, that $f, g$ are two mappings defined on the set $A$ with the values in $X$. If $f(A) \subseteq$ $g(A), g(A)$ is a complete subspace of $X$ and for all $x, y \in A$ :

(i) $d(f x, f y) \leq k d(g x, g y), 0 \leq k<1$ then $f$ and $g$ have a coincidence point, that is, there exists $z \in A$ such that $f(z)=g(z)$. 
The condition (i) of Theorem 1.1 appears to have been studied first by Machuca [6] in 1967 under some heavy topological conditions (see also [5, 7]).

It may be observed that the conclusion of Theorem 1.1 is not true (take $A=X$ ) if we drop completeness of $g(A)$. This can be seen by the following example.

Example 1.2 [8] Let $X=[2,20]$ and let $d$ be the usual metric on $X$. Define self-mappings $f$ and $g$ on $X$ as follows:

$$
\begin{aligned}
& f x=6 \quad \text { if } 2 \leq x \leq 5, \quad f x=\frac{x+5}{5} \quad \text { if } x>5, \\
& g x=12 \quad \text { if } 2 \leq x \leq 5, \quad g x=\frac{x+1}{3} \quad \text { if } x>5 .
\end{aligned}
$$

Then $f$ and $g$ satisfy the following conditions of Theorem 1.1, but they do not have a coincidence point:

(i) $f A=(2,5] \cup\{6\}, g A=(2,7] \cup\{12\}$ and $f A \subseteq g A$, for $A=X$;

(ii) $f$ and $g$ satisfy contraction condition $d(f x, f y) \leq \frac{3}{5} d(g x, g y)$.

Fixed point theorems are statements containing sufficient conditions that ensure the existence of a fixed point. Therefore, one of the central concerns in fixed point theory is to find a minimal set of sufficient conditions which guarantee a fixed point or a common fixed point as the case may be. Common fixed point theorems for contractive type mappings necessarily require a commutativity condition, a condition on the ranges of the mappings, continuity of one or more mappings besides a contractive condition. And every significant fixed point or common fixed point theorem attempts to weaken or obtain a necessary version of one or more of these conditions [9].

In 1976, using condition (i) of Theorem 1.1, Jungck [10] obtained common fixed point for commuting mappings by using a constructive procedure of sequence of iterates.

Theorem $1.3[10]$ Let $(X, d)$ be a complete metric space and let $f$ and $g$ be commuting self-maps of $X$ satisfying the conditions:

(i) $f X \subseteq g X$;

(ii) $d(f x, f y) \leq k d(g x, g y)$, for all $x, y \in X$ and some $0 \leq k<1$.

If $g$ is continuous then $f$ and $g$ have a unique common fixed point.

The essence of Jungck's theorem has been used by several workers to obtain interesting common fixed point theorems for both commuting and noncommuting pairs of mappings satisfying contractive type conditions. The constructive technique of Jungck's theorem has been further improved and extended by various researchers to establish common fixed point theorems for three mappings, four mappings and sequence of mappings (see also [11-17]).

Generalizations of Jungck's contraction condition have been extensively used to study common fixed points of contractive mappings. If $f$ and $g$ are two self-mappings of a metric space $(X, d)$, general contractive conditions assume the following form.

(a) $\phi$-type contractive condition (in the sense of Boyd and Wong [18]);

$$
d(f x, f y) \leq \phi(d(g x, g y))
$$


where $\phi: \mathbb{R}^{+} \rightarrow \mathbb{R}^{+}$is such that $\phi$ is upper semi-continuous from the right and $\phi(t)<t$ for each $t>0$.

(b) Given $\epsilon>0$ there exists a $\delta>0$ such that

$$
\epsilon \leq d(g x, g y)<\epsilon+\delta \Rightarrow d(f x, f y)<\epsilon
$$

Condition (b) is also referred to as a Meir-Keeler type $(\epsilon, \delta)$ contractive condition [19]. It can easily be seen that if $f$ and $g$ satisfy (b) then $f$ and $g$ also satisfy the contractive condition

$$
d(f x, f y)<d(g x, g y) .
$$

In some results the contractive condition (b) has been replaced by a slightly weaker contractive condition of the following form.

(c) Given $\epsilon>0$ there exists a $\delta>0$ such that

$$
\epsilon<d(g x, g y)<\epsilon+\delta \quad \Rightarrow \quad d(f x, f y) \leq \epsilon
$$

Jachymski [20] has shown that the contractive condition (c) implies (b) but not conversely.

In the setting of common fixed point theorems, the Meir-Keeler type $(\epsilon, \delta)$ contractive condition alone is not sufficient to guarantee the existence of a common fixed point. While assuming the $(\epsilon, \delta)$ contractive condition, the existence of a common fixed point is ensured either by imposing some additional restriction on $\delta$ or by assuming some additional condition besides the $(\epsilon, \delta)$ contractive condition or by imposing strong conditions on the continuity of mappings (for references see [9, 21-28]).

In 1982, Sessa gave the weaker version of the commutativity condition, namely the weakly commuting condition. In subsequent years Jungck [29, 30], Tivari and Singh [31], Pathak [32, 33], Jungck et al. [34], Jungck and Pathak [35], Pant [36], Pathak et al. [37], Al-Thagafi and Shahzad [38], Hussain et al. [39], Pant and Bisht [40], Bisht and Shahzad [41], and many others have considered several generalizations of commuting mappings or weaker notions of commutativity, see Table 1 . Now, it has been shown that weak compatibility is the minimal noncommuting condition for the existence of common fixed points of contractive type mapping pairs. In recent works several authors claimed to introduce some weaker noncommuting notions and showed that their introduced noncommuting conditions contain weak compatibility as a proper subclass. This is, however, of no use when searching for common fixed points. In fact most of the generalized commutativity notions fall in the subclass of weak compatibility in the setting of a unique common fixed point (or unique point of coincidence). These generalizations are novel but for their actual applications one should go beyond contractive conditions, since contractive conditions do not allow for more than one point of coincidence or fixed point.

In 2010, Haghi et al. [68] presented the following lemma, which is a consequence of the axiom of choice, and they showed that some coincidence point and common fixed point generalizations in fixed point theory are not real generalizations as they could easily be obtained from the corresponding fixed point theorems. Therefore, one should take care in obtaining real generalizations in fixed point theory (for more details see [68]). 
Table 1 Some weaker forms of commuting mappings

\begin{tabular}{|c|c|c|c|}
\hline S. No. & Forms & Year & References \\
\hline 1. & weakly commuting mappings & 1982 & Sessa $[42]$ \\
\hline 2. & asymptotically commuting mappings & 1986 & Tivari and Singh [31] \\
\hline 3. & compatible mappings & 1986 & Jungck [30] \\
\hline 4. & weakly* commuting mappings & 1986 & Pathak [32] \\
\hline 5. & weakly uniformly contraction mappings & 1990 & Pathak [43] \\
\hline 6. & weakly** commuting mappings & 1992 & Pathak [33] \\
\hline 7. & compatible mappings of type $(A)$ & 1993 & Jungck et al. [34] \\
\hline 8. & preorbitally commuting mappings & 1994 & Singh and Mishra [44] \\
\hline 9. & R-weakly commuting mappings & 1994 & Pant [36] \\
\hline 10. & pointwise $R$-weakly commuting mappings & 1994 & Pant [36] \\
\hline 11. & biased and weakly $g$-biased mappings & 1995 & Jungck and Pathak [35] \\
\hline 12. & semi-compatible mappings & 1995 & Cho et al. [45] \\
\hline 13. & compatible mappings of type $(B)$ & 1995 & Pathak and Khan [46] \\
\hline 14. & compatible mappings of type $(P)$ & 1995 & Pathak et al. [47] \\
\hline 15. & compatible mappings of type $(f)$ or type $(g)$ & 1995 & Pathak et al. [48] \\
\hline 16. & weakly compatible mappings & 1996 & Jungck [29] \\
\hline 17. & $f$-compatible or $g$-compatible mappings & 1997 & Pathak and Khan [49] \\
\hline 18. & $R$-weakly commuting mappings of type $\left(A_{f}\right)$ or $\left(A_{g}\right)$ & 1997 & Pathak et al. [50] \\
\hline 19. & $g$-biased mappings of type $(A)$ & 1998 & Pathak et al. [51] \\
\hline 20. & compatible mappings of type $(C)$ & 1998 & Pathak et al. [52] \\
\hline 21. & biased mappings of type $\left(A_{f}\right)$ or $\left(A_{g}\right)$ & 1999 & Fisher and Murthy [53] \\
\hline 22. & coincidentally commuting mappings & 1999 & Dhage [54] \\
\hline 23. & partially commuting mappings & 2000 & Sastry et al. [55] \\
\hline 24. & compatible mappings of type $(N)$ & 2000 & Srivastava et al. [56] \\
\hline 25. & intimate mappings & 2001 & Sahu et al. [57] \\
\hline 26. & compatible mappings of type $(C)$ & 2002 & Singh [58] \\
\hline 27. & weakly compatible mappings of type $(f)$ or type $(g)$ & 2007 & Pathak and Ume [59] \\
\hline 28. & occasionally weakly compatible mappings & 2008 & Al-Thagafi and Shahzad [38] \\
\hline 29. & Banach operator pairs & 2007 & Chen and Li [60] \\
\hline 30. & conditionally commuting mappings & 2009 & Pant and Pant [61] \\
\hline 31. & subcompatible mappings & 2009 & Bouhadjera and Godet-Thobie [62] \\
\hline 32. & P-operators & 2010 & Pathak and Hussain [63] \\
\hline 33. & almost compatible and almost biased mappings & 2010 & Suzuki and Pathak [64] \\
\hline 34. & $\phi$-weakly compatible of type $(f, g)$ & 2011 & Pathak and Tiwari [65] \\
\hline 35. & $J H$-operators & 2011 & Hussain et al. [39] \\
\hline 36. & conditionally compatible mappings & 2012 & Pant and Bisht [40] \\
\hline 37. & occasionally weakly biased pairs & $\begin{array}{l}2011 / \\
2012\end{array}$ & $\begin{array}{l}\text { Hussain et al. [39] and Bouhadjera and } \\
\text { Djoudi [66] }\end{array}$ \\
\hline 38. & pseudo compatible mappings & 2013 & Pant and Bisht [67] \\
\hline 39. & PD-operators & 2013 & Pathak and Rai [37] \\
\hline 40. & faintly compatible & 2013 & Bisht and Shahzad [41] \\
\hline
\end{tabular}

Lemma 1.4 [68] Let $X$ be a nonempty set and $f: X \rightarrow X$ a function. Then there exists a subset $E \subset X$ such that $f(E)=f(X)$ and $f: E \rightarrow X$ is one-to-one.

\section{Comparison of weaker forms of commuting mappings}

Let $f$ and $g$ be self-mappings of a set $X$. If $w=f x=g x$ for some $x$ in $X$, then $x$ is called a coincidence point of $f$ and $g$, and $w$ is called a point of coincidence (POC) of $f$ and $g$. The set of coincidence points $(C P)$ of $f$ and $g$ will be denoted by $C(f, g)$. Let $P C(f, g)$ represent the set of points of coincidence of $f$ and $g$. A point $x \in X$ is a common fixed point of $f$ and $g$ if $x=f x=g x$. The set of all common fixed points of $f$ and $g$ is denoted by $F(f, g)$.

Two self-mappings $f$ and $g$ of a metric space $(X, d)$ are said to be commuting iff $f g x=g f x$ for all $x$ in $X$.

The study of common fixed points of pair of self-mappings satisfying contractive type conditions becomes interesting in view of the fact that even commuting continuous map- 
pings on such nicely behaved entities as compact convex sets may fail to have a coincidence or common fixed point. When we extend such studies to the class of noncommuting contractive type mapping pair, it becomes still more interesting [36].

The first ever attempt to relax the commutativity of mappings to a smaller subset of the domain of mappings was initiated by Sessa [42] who in 1982 gave the notion of weak commutativity.

Definition 2.1 (Sessa [42]) Two self-mappings $f$ and $g$ of a metric space $(X, d)$ are called weakly commuting iff $d(f g x, g f x) \leq d(f x, g x)$ for all $x$ in $X$.

Notice that commuting mappings are obviously weakly commuting. However, a weakly commuting mappings need not be commuting.

Example 2.2 Let $X=[0,1]$ be equipped with the usual metric $d$ on $X$. Define constant mappings $f$ and $g: X \rightarrow X$ by

$$
f x=a \text { and } g x=b, \quad a \neq b .
$$

Then $f$ and $g$ are weakly commuting but not commuting since $d(f g x, g f x)=|a-b|=$ $d(f x, g x)$.

In order to enlarge the domain of noncommuting mappings, Pathak [32,33] obtained several new classes of noncommuting notions, namely, weak* commuting, weak ${ }^{* *}$ commuting mappings.

Definition 2.3 (Pathak [32]) Two self-mappings $f$ and $g$ of a metric space $(X, d)$ are called weak* commuting iff $d(f g x, g f x) \leq d\left(f^{2} x, g^{2} x\right)$ for all $x$ in $X$.

Definition 2.4 (Pathak [33]) Two self-mappings $f$ and $g$ of a metric space $(X, d)$ are called weak* $^{* *}$ commuting iff $f X \subset g X$ and, for any $x \in X$,

$$
d\left(f^{2} g^{2} x, g^{2} f^{2} x\right) \leq d\left(f^{2} g x, g f^{2} x\right) \leq d\left(g^{2} x, g^{2} f x\right) \leq d(f g x, g f x) \leq d\left(f^{2} x, g^{2} x\right) .
$$

It is easy to check that commuting mappings are weak* commuting and weak* commuting. The following example shows that the reverse implication does not hold.

Example 2.5 [32] Consider $X=[0,1]$ with the usual metric $d$ on $X$. Define $f, g: X \rightarrow X$ by

$$
f x=\frac{x}{x+3} \quad \text { for all } x \quad \text { and } \quad g x=\frac{x}{3} \quad \text { for all } x .
$$

Then $f$ and $g$ are weak* commuting and weak** commuting but $f$ and $g$ are not commuting mappings.

Remark 2.6 Notice that if $f^{2}=f$ and $g^{2}=g$, then weak* commutativity or weak ${ }^{* *}$ commutativity reduces to weak commutativity. 
Definition 2.7 (Pathak [43]) Two self-mappings $f$ and $g$ of a metric space $(X, d)$ are called weakly uniformly contraction mappings iff $d(f g x, g g x) \leq d(f x, g x)$ and $d(f f x, g f x) \leq d(f x, g x)$ for all $x$ in $X$.

In view of Example 2.5, we remark that commuting mappings are weakly uniformly contraction mappings. However, weakly uniformly contraction mappings need not be weakly commuting.

In 1986, Jungck generalized the concept of weak commutativity by introducing the notion of compatible mappings [30] also called asymptotically commuting mappings by Tivari and Singh [31] in an independent work. In [16] it has been shown that two continuous self-mappings of a compact metric space are compatible iff they commute on their set of coincidence points.

Definition 2.8 (Jungck [30], Tivari and Singh [31]) Two self-mappings $f$ and $g$ of a metric space $(X, d)$ are called compatible or asymptotically commuting iff $\lim _{n} d\left(f g x_{n}, g f x_{n}\right)=0$, whenever $\left\{x_{n}\right\}$ is a sequence in $X$ such that $\lim _{n} f x_{n}=\lim _{n} g x_{n}=t$ for some $t$ in $X$.

Clearly, weakly commuting mappings are compatible, but in view of Example 2.9 the converse does not hold.

Example 2.9 [30] Let $X=[0, \infty)$ and $d$ be the usual metric on $X$. Define $f, g: X \rightarrow X$ by

$$
f x=x^{3} \quad \text { for all } x \text { and } g x=2 x^{3} \quad \text { for all } x \text {. }
$$

Then $d(f g x, g f x)>d(f x, g x)$. Therefore $f$ and $g$ are not weakly commuting mappings. However, $f$ and $g$ are compatible mappings.

Remark 2.10 Notice that the notions of weak commutativity and compatibility differ in one respect. Weak commutativity is essentially a point property, while the notion of compatibility uses the machinery of sequences.

Remark 2.11 In a review of [30] Singh (Math. Rev. 89h:54030, see also [36]) has shown that for a pair of weakly commuting mappings on a metric space $(X, d)$, there may not exist even a single sequence $\left\{x_{n}\right\}$ in $X$ for which $\lim _{n} f x_{n}=\lim _{n} g x_{n}=t$ for some $t$ in $X$. In this case the mappings $f$ and $g$ are still compatible. The following example shows that in this situation they can be weakly commuting.

Example 2.12 Let $X=[0, \infty)$ and $d$ be the usual metric on $X$. Define $f, g: X \rightarrow X$ by

$$
f x=x \text { for all } x \text { and } g x=x+1 \text { for all } x \text {. }
$$

Then $f$ and $g$ are weakly commuting mappings but there does not exist even a single sequence for which the condition of compatibility is satisfied. However, $f g x=g f x=1+x$, i.e., $d(f g x, g f x)=0$. Hence $f$ and $g$ may be called vacuously compatible mappings.

Singh and Tomar [7] has also shown by an example that weak commutativity of a pair of self-mappings $f$ and $g$ on a metric space $(X, d)$ depends on the choice of the metric. This is true for compatibility as well. 
Example 2.13 [7] Let $X=[0, \infty)$ be endowed with the usual metric. Define $f, g: X \rightarrow X$ by

$$
f x=1+x \text { and } g x=2+x^{2} .
$$

Then $d(f g x, g f x)=2 x$ and $d(f x, g x)=\left(x^{2}-x+1\right)$. One may observe that $f$ and $g$ are not weakly commuting on $X$ with respect to the usual metric. But if $X$ is endowed with the discrete metric $d$, then $d(f g x, g f x)=1=d(f x, g x)$ for $x>0$. So, $f$ and $g$ are weakly commuting on $X$ when endowed with the discrete metric.

Ever since the introduction of compatibility, the study of common fixed points has developed around compatible maps and its weaker forms and it has become an area of vigorous research activity. However, fixed point theory for noncompatible mappings is equally interesting and Pant [69] has initiated some work along these lines. One can establish fixed point theorems for such mappings pairs not only under nonexpansive conditions but also under Lipschitz type conditions even without using the usual contractive method of proof. The best examples of noncompatible maps are found among pairs of mappings which are discontinuous at their common fixed point [69]. It may be observed that the mappings $f$ and $g$ are said to be noncompatible if there exists a sequence $\left\{x_{n}\right\}$ in $X$ such that for some $t$ in $X$ but $\lim _{n} d\left(f g x_{n}, g f x_{n}\right)$ is either non-zero or nonexistent.

Definition 2.14 [70] Two self-mappings $f$ and $g$ of a metric space $(X, d)$ are said to satisfy the (E.A.) property if there exists a sequence $\left\{x_{n}\right\}$ in $X$ such that

$$
\lim _{n \rightarrow \infty} f x_{n}=\lim _{n \rightarrow \infty} g x_{n}=t \quad \text { for some } t \in X
$$

It may be observed that the (E.A.) property is equivalent to the previously known notion of tangential mappings introduced by Sastry et al. [55].

If $f$ and $g$ are both noncompatible then they do satisfy the (E.A.) property. In fact the notion of the (E.A.) property circumvents the most crucial part of fixed point theorems consisting of constructive procedures yielding a Cauchy sequence. On the other hand the (E.A.) property enables us to study the existence of common fixed point of nonexpansive or Lipschitz type conditions in the setting of noncomplete metric spaces.

Sintunavarat and Kumam [71] introduced an interesting property, namely the common limit in the range property (in short $C L R_{g}$ ) which completely buys the condition of closedness of the ranges of the involved mappings and has an edge over the (E.A.) property (see also [72-77]).

Theorem $2.15[71,78]$ Two self-mappings $f$ and $g$ of a metric space $(X, d)$ are said to be satisfy the common limit in the range of $g$ property (in short $C L R_{g}$ ) if there exists a sequence $\left\{x_{n}\right\}$ in $X$ such that

$$
\lim _{n \rightarrow \infty} f x_{n}=\lim _{n \rightarrow \infty} g x_{n}=g x \quad \text { for some } x \in X .
$$

It is important to note that in the setting of metric spaces, there is no general method for the study of common fixed points of nonexpansive or Lipschitz type mappings. The 
notions of noncompatibility, the (E.A.) property and $\left(C L R_{g}\right)$ property are well suited for studying common fixed points of strict contractive conditions, nonexpansive type mapping pairs or Lipschitz type mapping pairs in ordinary metric spaces, which are not even complete.

Definition 2.16 (Singh and Mishra [44]) If for $x_{0} \in X$ there exists a sequence $\left\{x_{n}\right\}$ in $X$ such that $f x_{n+1}=g x_{n}, n=0,1,2, \ldots$, then $O\left(g, f ; x_{0}\right)=\left\{x_{n}: n=0,1,2, \ldots\right\}$ is an orbit for $g$ and $f$. Maps $f$ and $g$ are weakly $x_{0}$-preorbitally commuting iff there exists a positive integer $N$ such that $d\left(f g x_{n}, g f x_{n}\right) \leq d\left(f x_{n}, g x_{n}\right)$ for every $\left\{x_{n}\right\}(n \geq N)$ occurring in $O\left(g, f ; x_{0}\right)$.

Weakly commuting mappings are preorbitally commuting but the converse is not true in general.

Example 2.17 [79] Let $X=[0, \infty)$ and $d$ be the usual metric on $X$. Define $f, g: X \rightarrow X$ by

$$
f x=3 x^{3} \quad \text { for all } x \quad \text { and } \quad g x=x^{3} \quad \text { for all } x .
$$

Then $d(f g x, g f x)>d(f x, g x)$, i.e., $f$ and $g$ are not weakly commuting but $f$ and $g$ are preorbitally commuting mappings (e.g., take $\left\{x_{n}\right\}=0$ ).

Definition 2.18 Two self-mappings $f$ and $g$ of a metric space $(X, d)$ are called:

(i) compatible of type (A) (Jungck et al. [34]) iff

$$
\lim _{n} d\left(f f x_{n}, g f x_{n}\right)=0 \quad \text { and } \quad \lim _{n} d\left(f g x_{n}, g g x_{n}\right)=0
$$

(ii) compatible of type (B) (Pathak et al. [46]) iff

$$
\lim _{n} d\left(f g x_{n}, g g x_{n}\right) \leq \frac{1}{2}\left[\lim _{n} d\left(f g x_{n}, f t\right)+\lim _{n} d\left(f t, f x_{n}\right)\right]
$$

and

$$
\lim _{n} d\left(g f x_{n}, f f x_{n}\right) \leq \frac{1}{2}\left[\lim _{n} d\left(g f x_{n}, g t\right)+\lim _{n} d\left(g t, g g x_{n}\right)\right],
$$

(iii) compatible of type (C) (Pathak et al. [52]) iff

$$
\lim _{n} d\left(f g x_{n}, g g x_{n}\right) \leq \frac{1}{3}\left[\lim _{n} d\left(f g x_{n}, f t\right)+\lim _{n} d\left(f t, f f x_{n}\right)+\lim _{n} d\left(f t, g g x_{n}\right)\right]
$$

and

$$
\lim _{n} d\left(g f x_{n}, f f x_{n}\right) \leq \frac{1}{3}\left[\lim _{n} d\left(g f x_{n}, g t\right)+\lim _{n} d\left(g t, g g x_{n}\right)+\lim _{n} d\left(g t, f f x_{n}\right)\right],
$$

whenever $\left\{x_{n}\right\}$ is a sequence in $X$ such that $\lim _{n} f x_{n}=\lim _{n} g x_{n}=t$ for some $t$ in $X$.

Proposition 2.19 [80] Let $f$ and $g$ be continuous mappings from a metric space $(X, d)$ into itself. Then the following are equivalent:

(i) $f$ and $g$ are compatible of type $(A)$, 
(ii) $f$ and $g$ are compatible of type $(B)$,

(iii) $f$ and $g$ are compatible of type $(C)$,

(iv) $f$ and $g$ are compatible.

It is clear to see that compatible mappings of type $(A) \Longrightarrow$ compatible of type $(B) \Longrightarrow$ compatible of type $(C)$, but the converse is not true in general.

Example 2.20 [80] Let $X=[1,20]$ and $d$ be the usual metric on $X$. Define $f, g: X \rightarrow X$ as follows:

$$
\begin{aligned}
& f x=1 \quad \text { if } x=1, \quad f x=3 \quad \text { if } 1<x \leq 7, \quad g x=x-6 \quad \text { if } 7<x \leq 20, \\
& g x=1 \quad \text { if } x \in\{1\} \cup(7,20], \quad g x=2 \quad \text { if } 1<x \leq 7 .
\end{aligned}
$$

It may be observed that $f$ and $g$ are compatible of type $(C)$ but neither compatible nor compatible of type $(A)$ nor compatible of type $(B)$ (consider the sequence $\left\{x_{n}\right\}$ given by $\left.x_{n}=7+\frac{1}{n}: n>0\right)$.

Example 2.21 [8] Let $X=[2,12]$ and $d$ be the usual metric on $X$. Define $f, g: X \rightarrow X$ as follows:

$$
\begin{aligned}
& f x=2 \quad \text { if } x=2 \text { or } x>5, \quad f x=12 \quad \text { if } 2<x \leq 5, \\
& g 2=2, \quad g x=12 \quad \text { if } 2<x \leq 5, \quad g x=\frac{x+1}{3} \quad \text { if } x>5 .
\end{aligned}
$$

It may be observed that $f$ and $g$ are compatible mappings of type (A), but neither commuting nor compatible mappings. To see this let us consider the sequence $\left\{x_{n}\right\}$ given by $x_{n}=$ $5+\frac{1}{n}: n>0$. Then $f x_{n} \rightarrow 2, g x_{n} \rightarrow 2, f g x_{n}=g g x_{n} \rightarrow 12, g f x_{n}=f f x_{n} \rightarrow 2, \lim _{n} d\left(f g x_{n}, g g x_{n}\right)=$ $\lim _{n} d\left(f f x_{n}, g f x_{n}\right)=0$ and $\lim _{n} d\left(f g x_{n}, g f x_{n}\right)=\lim _{n} d\left(f x_{n}, g g x_{n}\right) \neq 0$.

Examples 2.21 and 2.22 (below) show that the notions of compatible mappings and compatible of type $(A)$ are independent to each other.

Example 2.22 [81] Let $X=\mathbb{R}$ equipped with the usual metric $d$. Define self-mappings $f$ and $g$ as follows:

$$
f(x)=x \quad \text { and } \quad g(x)= \begin{cases}0 & \text { if } x \text { is an integer } \\ 1 & \text { if } x \text { is not an integer }\end{cases}
$$

Then for the sequence $\left\{x_{n}\right\}=1+\frac{1}{n+1}$ we get $\lim _{n} f x_{n}=\lim _{n} g x_{n}=1, \lim _{n} d\left(f g x_{n}, g f x_{n}\right)=0$, but $\lim _{n} d\left(f f x_{n}, g f x_{n}\right) \neq 0, \lim _{n} d\left(g f x_{n}, g g x_{n}\right) \neq 0$ and $\lim _{n} d\left(f f x_{n}, g g x_{n}\right) \neq 0$. Therefore $f$ and $g$ are compatible but not compatible of type $(A)$.

Definition 2.23 (Pant [36]) Two self-mappings $f$ and $g$ of a metric space $(X, d)$ are called $R$-weakly commuting iff there exists some positive real number $R$ such that $d(f g x, g f x) \leq$ $R d(f x, g x)$ for all $x$ in $X$.

Notice that weak commutativity of a pair of self-mappings implies their $R$-weak commutativity and the converse is true only when $R \leq 1$. 
Example 2.24 [36] Let $X=[1, \infty)$ be endowed with the usual metric. Define $f, g: X \rightarrow X$ by

$$
f x=2 x-1 \quad \text { and } g x=x^{2} \quad \text { for all } x \in X
$$

Then $d(f g x, g f x)=2 d(f x, g x)$. Thus $f$ and $g$ are $R$-weakly commuting $(R=2)$ but are not weakly commuting.

Definition 2.25 (Pathak et al. [50]) Two self-mappings $f$ and $g$ of a metric space $(X, d)$ are called:

(i) $R$-weakly commuting of type $\left(A_{f}\right)$ iff there exists some positive real number $R$ such that $d(f g x, g g x) \leq R d(f x, g x)$ for all $x$ in $X$.

(ii) $R$-weakly commuting of type $\left(A_{g}\right)$ iff there exists some positive real number $R$ such that $d(f f x, g f x) \leq R d(f x, g x)$ for all $x$ in $X$.

It may be observed that definition (ii) can be obtained from definition (i) by interchanging the role of $f$ and $g$. Further, $R$-weakly commuting pair of self-mappings are independent of $R$-weakly commuting of type $\left(A_{f}\right)$ or type $\left(A_{g}\right)$. Example 2.24 shows that $d(f g x, g g x)>R d(f x, g x)$ for each $x>1$ and some $R>0(e . g$., say $R=4)$. Thus $f$ and $g$ are $R$-weakly commuting but not $R$-weakly commuting of type $\left(A_{f}\right)$.

The next example shows that $f$ and $g$ are $R$-weakly commuting of type $\left(A_{f}\right)$ but not $R$-weakly commuting mappings.

Example 2.26 [50] Let $X=[-1,2]$ equipped with the usual metric $d$. Define selfmappings $f$ and $g$ on $X$ as follows:

$$
f(x)=\left\{\begin{array}{ll}
1 & \text { if } x \in[-1,1], \\
\frac{3}{4} & \text { if } x \in\left(1, \frac{5}{4}\right), \\
1+\frac{1}{32} x^{2} & \text { if } x \in\left[\frac{5}{4}, 2\right],
\end{array} \quad g(x)= \begin{cases}1+\frac{1}{4} x^{2} & \text { if } x \in[-1,1) \\
1 & \text { if } x=1, \\
2 & \text { if } x \in\left(1, \frac{5}{4}\right) \\
1-\frac{1}{8} x^{2} & \text { if } x \in\left[\frac{5}{4}, 2\right]\end{cases}\right.
$$

In this example $f$ and $g$ are $R$-weakly commuting pair $\left(A_{f}\right)$ for $R=6$ but not $R$-weakly commuting mappings [50]. Thus $R$-weakly commuting mappings and $R$-weakly commuting of $\left(A_{f}\right)$ or $\left(A_{g}\right)$ mappings are independent to each other.

It may be noted that both compatible and noncompatible mappings can be $R$-weakly commuting of type $\left(A_{g}\right)$ or $\left(A_{f}\right)$.

Example 2.27 [82] Let $X=[1, \infty)$ be endowed with the usual metric. Define $f, g: X \rightarrow X$ by

$$
f x=2 x-1 \text { and } g x=3 x-2 \text { for all } x \in X .
$$

Then it can be verified in this example that $f$ and $g$ are compatible. Furthermore, $f$ and $g$ are $R$-weakly commuting of type $\left(A_{f}\right)$ with $R=3$ and $R$-weakly commuting of type $\left(A_{g}\right)$ with $R=2$. 
Example 2.28 [8] Let $X=[2,20]$ and $d$ be the usual metric on $X$. Define $f, g: X \rightarrow X$ as follows:

$$
\begin{aligned}
& f x=2 \quad \text { if } x=2 \text { or } x>5, \quad f x=12 \quad \text { if } 2<x \leq 5, \\
& g 2=2, \quad g x=\frac{x+31}{3} \quad \text { if } 2<x \leq 5, \quad g x=\frac{x+1}{3} \quad \text { if } x>5 .
\end{aligned}
$$

It may be observed that $f$ and $g$ are $R$-weakly commuting of type $\left(A_{g}\right)$ since $d(g f x, g g x) \leq$ $d(f x, g x)$ for all $x \in X$. To see that $f$ and $g$ are noncompatible, let us consider a sequence $\left\{x_{n}\right\}$ given by $x_{n}=5+\frac{1}{n}: n>0$. Then $f x_{n} \rightarrow 2, g x_{n} \rightarrow 2, f g x_{n} \rightarrow 12, g f x_{n} \rightarrow 2$, and $\lim _{n} d\left(f g x_{n}, g f x_{n}\right) \neq 0$.

Definition 2.29 [83] Two self-mappings $f$ and $g$ of a metric space $(X, d)$ are called $R$-weakly commuting of type $(P)$ iff there exists some positive real number $R$ such that $d(f x, g g x) \leq R d(f x, g x)$ for all $x$ in $X$.

The next example depicts when two mappings representing parallel straight lines on the real plane shall be commuting, weakly commuting, $R$-weakly commuting or analogous definitions of $R$-weakly commuting mappings.

Example 2.30 [9] Let $X=\mathbb{R}$ equipped with the usual metric $d$. Define self-mappings $f$ and $g$ as follows:

$$
f x=m x+a \quad \text { and } g x=m x+b, \quad a \neq b .
$$

Then $d(f g x, g f x)=d(f f x, g g x)=|m-1| \cdot|a-b|, d(f f x, g f x)=d(f g x, g g x)=|a-b|$ and $d(f x, g x)=$ $|a-b|$. Thus $f$ and $g$ will be:

(i) commuting if $m=1$,

(ii) weakly commuting if $0 \leq m \leq 2$,

(iii) $R$-weakly commuting or $R$-weakly commuting of type $(P)$ if $|m-1| \geq 1$.

Remark 2.31 If $f$ and $g$ are $R$-weakly commuting or $R$-weakly commuting $\left(A_{f}\right)$ or $R$ weakly commuting of type $\left(A_{g}\right)$ or $R$-weakly commuting $(P)$ and if $z$ is their coincidence point, i.e., $f z=g z$, then we get $f f z=f g z=g f z=g g z$. Thus at a coincidence point, all the analogous notions of $R$-weak commutativity including $R$-weak commutativity are equivalent to each other and imply their commutativity.

Definition 2.32 (Jungck and Pathak [35]) Two self-mappings $f$ and $g$ of a metric space $(X, d)$ are called $f$-biased iff

$$
\alpha \lim _{n} d\left(f x_{n}, f g x_{n}\right) \leq \alpha \lim _{n} d\left(g x_{n}, g f x_{n}\right),
$$

where $\alpha \lim _{n}$ stays for $\limsup \operatorname{sun}_{n}$ or $\liminf _{n}$, whenever $\left\{x_{n}\right\}$ is a sequence in $X$ such that $\lim _{n} f x_{n}=\lim _{n} g x_{n}=t$ for some $t$ in $X$.

Similarly, the definition of $g$-biased can be obtained from the definition of $f$-biased by interchanging the role of $f$ and $g$. 
Jungck and Pathak [35] have shown that if $f$ and $g$ are compatible then they are both $f$-biased and $g$-biased, but the converse is not true.

Example 2.33 Let $X=[0,1]$ and $d$ be the usual metric on $X$. Define $f, g: X \rightarrow X$ by

$$
\begin{aligned}
& f x=1-2 x, \quad g x=2 x \quad \text { for } x \in\left[0, \frac{1}{2}\right], \\
& f x=0, \quad g x=1 \quad \text { for } x \in\left(\frac{1}{2}, 1\right] .
\end{aligned}
$$

Then $f$ and $g$ are both $f$-biased and $g$-biased but not compatible.

Definition 2.34 (Jungck and Pathak [35]) Two self-mappings $f$ and $g$ of a metric space $(X, d)$ are called weakly $f$-biased iff

$$
f x=g x \quad \text { implies } \quad d(f g x, f x) \leq d(g f x, g x) .
$$

Clearly, every biased mappings are weakly biased mappings (see Proposition 1.1 in [35]) but the converse is false in general.

Sahu et al. [57] have shown that intimate mappings are more general than compatible mappings.

Definition 2.35 (Sahu et al. [57]) Two self-mappings $f$ and $g$ of a metric space $(X, d)$ are called $f$-intimate iff

$$
\alpha \lim _{n} d\left(f x_{n}, f g x_{n}\right) \leq \alpha \lim _{n} d\left(g x_{n}, g g x_{n}\right),
$$

where $\alpha \lim _{n}$ stays for $\limsup \operatorname{sun}_{n}$ or $\liminf _{n}$, whenever $\left\{x_{n}\right\}$ is a sequence in $X$ such that $\lim _{n} f x_{n}=\lim _{n} g x_{n}=t$ for some $t$ in $X$.

Next example [57] shows that intimate mappings need not be compatible.

Example 2.36 Let $X=[0,1]$ and $d$ be the usual metric on $X$. Define $f, g: X \rightarrow X$ as follows:

$$
f x=\frac{2}{x+2} \quad \text { and } \quad g x=\frac{1}{1+x} \quad \text { for } x \in[0,1] .
$$

For this let us consider the sequence $\left\{x_{n}\right\}$ given by $x_{n}=\frac{1}{n}: n>0$. Then $f x_{n} \rightarrow 1, g x_{n} \rightarrow 1$ and $\lim _{n} d\left(f g x_{n}, f x_{n}\right)<\lim _{n} d\left(g g x_{n}, g x_{n}\right)$, i.e., $f$ and $g$ are $f$-intimate but $\lim _{n} d\left(f g x_{n}, g f x_{n}\right) \neq 0$.

Definition 2.37 (Cho et al. [45]) Two self-mappings $f$ and $g$ of a metric space $(X, d)$ are called semi-compatible iff

(i) $f t=g t$ implies $f g t=g f t$;

(ii) $\lim _{n} f x_{n}=\lim _{n} g x_{n}=t$ for some $t$ in $X$ implies $\lim _{n} d\left(f g x_{n}, g t\right)=0$.

It may be noted that semi-compatible mappings need not be compatible mappings. 
Example 2.38 [9] Let $X=[2,6]$ and $d$ be the usual metric on $X$. Define $f, g: X \rightarrow X$ as follows:

$$
\begin{aligned}
& f x=2 \quad \text { if } x<3, \quad f x=4 \quad \text { if } x=3, \quad f x=\frac{x+21}{12} \quad \text { if } 3<x \leq 6, \\
& g 2=2, \quad g x=2 x \quad \text { if } 2<x \leq 3, \quad g x=\frac{2 x}{3} \quad \text { if } 3<x \leq 6 .
\end{aligned}
$$

Then $f$ and $g$ are semi-compatible, but noncompatible mappings. To see this let us consider a decreasing sequence $\left\{x_{n}\right\}$ given by $3<x_{n}<4$ and $\lim _{n} x_{n}=3$. Then $f x_{n} \rightarrow 2$, $g x_{n} \rightarrow 2, f g x_{n} \rightarrow 2=g 2, g f x_{n} \rightarrow 4, \lim _{n} d\left(f g x_{n}, g t\right)=0, f g 2=g f 2$ and $\lim _{n} d\left(f g x_{n}, g f x_{n}\right) \neq 0$.

In 1997, Pathak et al. [49] weakened the notion of compatible of type $(A)$ by splitting it into two parts, namely $f$-compatible and $g$-compatible.

Definition 2.39 Two self-mappings $f$ and $g$ of a metric space $(X, d)$ are called $f$-compatible (Pathak et al. [49]) iff

$$
\lim _{n} d\left(f g x_{n}, g g x_{n}\right)=0
$$

whenever $\left\{x_{n}\right\}$ is a sequence in $X$ such that $\lim _{n} f x_{n}=\lim _{n} g x_{n}=t$ for some $t$ in $X$.

The definition of $g$-compatible can be obtained from the definition of $f$-compatible by interchanging the role of $f$ and $g$.

The following propositions have been proved in [49].

Proposition 2.40 [49] Let $f, g:(X, d) \rightarrow(X, d)$ be mappings with $g$ continuous. Then $f$ and $g$ are compatible iff they are $f$-compatible.

Proposition 2.41 [49] Let $f$ and $g$ be continuous mappings from a metric space $(X, d)$ into itself. Then the following are equivalent:

(i) $f$ and $g$ are compatible,

(ii) $f$ and $g$ are $f$-compatible,

(iii) $f$ and $g$ are $g$-compatible.

If $f$ and $g$ are discontinuous mappings then the concepts compatible, $f$-compatible, g-compatible are independent to each others.

Example 2.42 [49] Let $X=\mathbb{R}$ and $d$ be the usual metric on $X$. Define $f, g: X \rightarrow X$ as follows:

$$
\begin{aligned}
& f x=\frac{1}{x^{3}} \quad \text { if } x \neq 0, \quad f x=2 \quad \text { if } x=0, \\
& g x=\frac{1}{x^{2}} \quad \text { if } x \neq 0, \quad g x=1 \quad \text { if } x=0 .
\end{aligned}
$$

Then $f$ and $g$ are compatible but not $f$-compatible nor $g$-compatible. To see that, let us consider a sequence $\left\{x_{n}\right\}$ given by $x_{n}=n$. Then $f x_{n} \rightarrow 0, g x_{n} \rightarrow 0, \lim _{n} d\left(f g x_{n}, g f x_{n}\right)=0$ but $\lim _{n} d\left(f g x_{n}, g f x_{n}\right)=\lim _{n} d\left(f g x_{n}, g f x_{n}\right)=\infty$, as $n \rightarrow \infty$. 
Example 2.43 [49] Let $X=[0,1]$ equipped with the usual metric $d$. Define self-mappings $f$ and $g$ as follows:

$$
f(x)=x \text { and } g(x)= \begin{cases}1-x & \text { if } 0 \leq x<\frac{1}{2} \\ 1 & \text { if } \frac{1}{2} \leq x \leq 1\end{cases}
$$

Then $f$ and $g$ are compatible and $g$-compatible but not $f$-compatible.

Example 2.44 [49] Let $X=[0, \infty)$ and $d$ be the usual metric on $X$. Define $f, g: X \rightarrow X$ as follows:

$$
\begin{aligned}
& f x=x \quad \text { if } 0 \leq x<1, \quad f x=2 \quad \text { if } 1 \leq x<\infty, \\
& g x=2-x \quad \text { if } 0 \leq x<1, \quad f x=2 \quad \text { if } 1 \leq x<\infty .
\end{aligned}
$$

Then $f$ and $g$ are both $f$-compatible and $g$-compatible but not compatible.

Definition 2.45 Two self-mappings $f$ and $g$ of a metric space $(X, d)$ are called:

(i) compatible of type $(P)$ (Pathak et al. $[47,84,85])$ iff

$$
\lim _{n} d\left(f f x_{n}, g g x_{n}\right)=0
$$

(ii) compatible of type (C) (Singh [58]) iff

$$
\lim _{n} d\left(f f x_{n}, g g x_{n}\right)=0 \text { and } \lim _{n} d\left(f g x_{n}, g f x_{n}\right)=0,
$$

whenever $\left\{x_{n}\right\}$ is a sequence in $X$ such that $\lim _{n} f x_{n}=\lim _{n} g x_{n}=t$ for some $t$ in $X$.

Proposition 2.46 Let $f, g:(X, d) \rightarrow(X, d)$ be mappings. If $f$ and $g$ are either compatible or compatible of type $(A)$ or $f$-compatible, or g-compatible or compatible of type $(P)$ or compatible of type $(C)$ and $f z=g z$ for some $z \in X$ then $f f z=f g z=g f z=g g z$.

Proof Let $\left\{x_{n}\right\}$ be a sequence in $X$ defined by $x_{n}=z, n \in \mathbb{N}$ and $f z=g z$ for some $z \in X$. Then we have $\lim _{n} f x_{n}=\lim _{n} g x_{n}=f z=g z$. Since $f$ and $g$ are either compatible or compatible of type $(A)$ or $f$-compatible, or $g$-compatible or compatible of type $(P)$ or compatible of type $(C)$, we have $d(f g z, g f z)=\lim _{n} d\left(f g x_{n}, g f x_{n}\right)=0$ or $d(f f z, g f z)=\lim _{n} d\left(f f x_{n}, g g x_{n}\right)=$ $0=d(g g z, g f z)=\lim _{n} d\left(g g x_{n}, g f x_{n}\right)$ or $d(f f z, g f z)=\lim _{n} d\left(f f x_{n}, g g x_{n}\right)=0$. Therefore, $f f z=f g z=$ $g f z=g g z$.

Definition 2.47 Two self-mappings $f$ and $g$ of a metric space $(X, d)$ are called compatible mappings of type $(f)$ (Pathak et al. [48]) iff

$$
\lim _{n} d\left(g f x_{n}, g x_{n}\right)+\lim _{n} d\left(f g x_{n}, g f x_{n}\right)=\lim _{n} d\left(f g x_{n}, f x_{n}\right),
$$

whenever $\left\{x_{n}\right\}$ is a sequence in $X$ such that $\lim _{n} f x_{n}=\lim _{n} g x_{n}=t$ for some $t$ in $X$.

In 1996, Jungck generalized the notion of compatible mappings. 
Definition 2.48 (Jungck [29], Sastry et al. [55] and Dhage [54], Shrivastava et al. [56]) Two self-maps $f$ and $g$ of a metric space $(X, d)$ are called weakly compatible (partially commuting or coincidentally commuting, compatible type $(N))$ iff $f$ and $g$ commute on the set of coincidence points.

In 1998, Pant investigated existence of common fixed points for noncompatible mappings and pointwise $R$-weak commutativity, which he defined in 1994 [36] without giving any name.

Definition 2.49 (Pant [86]) Two self-mappings $f$ and $g$ are called pointwise $R$-weakly commuting on $X$ iff given $x$ in $X$ there exists $R>0$ such that $d(f g x, g f x) \leq R d(f x, g x)$.

It is obvious from the definition that $f$ and $g$ can fail to be pointwise $R$-weakly commuting only if there exists some $x$ in $X$ such that $f x=g x$ while $f g x \neq g f x$, i.e., only if they posses a coincidence point at which they do not commute.

Remark 2.50 [9] Compatible mappings are pointwise $R$-weakly commuting. To see this, let $f x=g x$. Consider the constant sequence $\left\{x_{n}: x_{n}=x\right\}$. Then $\lim _{n} f x_{n}=f x=g x=$ $\lim _{n} g x_{n}=t$. Compatibility of $f$ and $g$ implies that $\lim _{n} d\left(f g x_{n}, g f x_{n}\right)=0$, i.e., $d(g f x, f g x)=0$ or $f g x=g f x$. However, pointwise $R$-weakly commuting mappings need not be compatible (see Example 2.52).

Remark 2.51 [9] Pointwise $R$-weak commutativity is a necessary condition for the existence of common fixed points of contractive type mapping pairs. Suppose $f$ and $g$ be a contractive type pair of self-mappings of a metric space $(X, d)$ having a common fixed point, say $z$ then $z=f z=g z$ and $f g z=g f z=f z=g z=z$. If possible, suppose that $f$ and $g$ are not pointwise $R$-weakly commuting. Then there exists a point $w$ in $X$ such that $f w=g w$ while $f g w \neq g f w$. We thus have $f w=g w$ and $f z=g z$ with $f w \neq f z$. This is not possible in view of contractive conditions. For example, if $f$ and $g$ satisfy the contractive condition

$d(f x, f y)<\max \{d(g x, g y), d(f x, g x), d(f y, g y), d(f x, g y), d(f y, g x)\}$, which is one of the most general contractive conditions, then we get

$d(f w, f z)<\max \{d(g w, g z), d(f w, g w), d(f z, g z), d(f w, g z), d(f z, g w)\}=d(f w, f z)$, a contradiction. This shows that existence of a common fixed point satisfying contractive conditions implies pointwise $R$-weakly commuting.

(It is also well known that pointwise $R$-weak commutativity is equivalent to commutativity at coincidence points and in the setting of metric spaces this notion is equivalent to weak compatibility.)

If $f$ and $g$ are compatible or $f$-compatible or $g$-compatible or compatible of type ( $A$ ) then they are obviously weakly compatible but as shown in Example 2.52 converse is not true.

Example 2.52 Let $X=\mathbb{R}$ equipped with the usual metric $d$. Define self-mappings $f$ and $g$ as follows:

$$
f(x)=[x] \text { and } g(x)= \begin{cases}-1 & \text { if } x \leq 0 \\ 0 & \text { if } 0<x<2 \\ 1 & \text { if } x \geq 2\end{cases}
$$

where $[x]$ denotes the integral part of $x$. 
Then for the sequence $\left\{x_{n}\right\}=\frac{1}{n}$ we get $\lim _{n} f x_{n}=\lim _{n} g x_{n}=0$ and $\lim _{n} d\left(f g x_{n}, g f x_{n}\right) \neq 0$, $\lim _{n} d\left(f f x_{n}, g f x_{n}\right) \neq 0$ and $\lim _{n} d\left(g f x_{n}, g g x_{n}\right) \neq 0$. Therefore $f$ and $g$ are neither compatible nor $f$-compatible nor $g$-compatible nor compatible of type $(A)$ nor compatible of type $(P)$ nor compatible of type $(C)$ but they are weakly compatible as they commute at their coincidence points $x=-1,1$.

In order to obtain new common fixed point theorems, one should be careful to use nontrivial noncommuting conditions. For example see the following result.

In [87] the authors obtained Corollary 2.53 as a particular case of their main theorem (see Theorem 3.1 in [87] and take $A=f$ and $S=g$ ):

Corollary 2.53 Let $f$ and $g$ be weakly compatible self-mappings of a complete metric space $(X, d)$ satisfying

(i) $f X \subseteq g X$;

(ii) $d(f x, f y) \leq k d(g x, g y)$, for all $x, y \in X$ and some $0 \leq k<1$.

Then $f$ and $g$ have a unique common fixed point in $X$.

In some cases the condition of completeness mentioned in the above corollary may be replaced by the (E.A.) property besides some condition on the ranges of the involved mappings $[55,70]$.

Corollary 2.54 Let $f$ and $g$ be weakly compatible self-mappings of a metric space $(X, d)$ satisfying the (E.A.) property, and

(i) $f X \subseteq g X$;

(ii) $d(f x, f y) \leq k d(g x, g y)$, for all $x, y \in X$ and some $0 \leq k<1$.

If the range off or $g$ is a complete subspace of $X$ then $f$ and $g$ have $a$ unique common fixed point in $X$.

It may be observed that the conclusions of above corollaries are not true. This can be seen from the following counter example [8].

Example 2.55 [8] Let $X=[2,20]$ and $d$ be the usual metric on $X$. Define self-mappings $f$ and $g$ on $X$ as follows:

$$
\begin{array}{ll}
f x=6 \quad \text { if } 2 \leq x \leq 5, & f x=\frac{x+5}{5} \quad \text { if } x>5, \\
g x=12 & \text { if } 2 \leq x \leq 5, \quad g x=\frac{x+1}{3} \quad \text { if } x>5 .
\end{array}
$$

Then $f$ and $g$ satisfy the following conditions of above corollaries, respectively, but do not have a common fixed point.

(i) $f X=(2,5] \cup\{6\}, g X=(2,7] \cup\{12\}$ and $f X \subseteq g X$;

(ii) $f$ and $g$ satisfy a particular contraction condition $d(f x, f y) \leq \frac{3}{5} d(g x, g y)$;

(iii) $f$ and $g$ are trivially weakly compatible;

(iv) $f$ and $g$ are also tangential mappings. To see this let $\left\{x_{n}\right\}$ be the sequence in $X$ given by $x_{n}=5+\epsilon_{n}$ where $\epsilon_{n} \rightarrow 0$ as $n \rightarrow \infty$. Then $\lim _{n \rightarrow \infty} f x_{n}=\lim _{n \rightarrow \infty} g x_{n}=2$.

One can see in Example 2.55 (above) that both the mappings $f$ and $g$ satisfy weak compatibility condition vacuously, yet $f$ and $g$ are common fixed point free mappings. We can redefine the notion of weak compatible mappings in the following way. 
Definition 2.56 Two self-mappings $f$ and $g$ of a metric space $(X, d)$ are called nontrivially weakly compatible if they commute on the set of coincidence points whenever the set of their coincidences is nonempty.

Definition 2.57 (Pathak et al. [88]) Two self-mappings $f$ and $g$ of a metric space $(X, d)$ are called compatible of type $(I)$ iff

$$
d(t, g t) \leq \lim \sup d\left(t, f g x_{n}\right)
$$

whenever $\left\{x_{n}\right\}$ is a sequence in $X$ such that $\lim _{n} f x_{n}=\lim _{n} g x_{n}=t$ for some $t$ in $X$.

The following examples show that weakly compatible mappings and compatible mappings of type $(I)$ are independent from each other.

Example 2.58 [88] Let $X=[0, \infty)$ and $d$ be the usual metric on $X$. Define $f, g: X \rightarrow X$ by

$$
f x= \begin{cases}\cos x & \text { if } x \neq 1 \\ 0 & \text { if } x=1\end{cases}
$$

and

$$
g x= \begin{cases}e^{x} & \text { if } x \neq 1 \\ 0 & \text { if } x=1\end{cases}
$$

Then $f x=g x$ iff $x=0$ and $x=1$. Also at these points $f g x=g f x$. It means the mappings $f$ and $g$ are weakly compatible. It can also be noted that $f$ and $g$ not compatible of type (I). To see this, let $\left\{x_{n}\right\}$ be a sequence in $X$ such that $f x_{n} \rightarrow t, g x_{n} \rightarrow t$. Then for $t=1, d(t, g t)>$ $d\left(t, f g x_{n}\right)$.

Example 2.59 [88] Let $X=[0,1)$ be endowed with the usual metric. Define $f, g: X \rightarrow X$ by

$$
f x=2 x+1 \text { and } g x=x^{2}+1 \quad \text { for all } x \in X .
$$

Then at $x=0, f x=g x$. But $f g x \neq g f x$, which shows that $f$ and $g$ are not weakly compatible but compatible of type $(I)$. To see this, let $\left\{x_{n}\right\}$ be a sequence in $X$ such that $f x_{n} \rightarrow t, g x_{n} \rightarrow t$. Then for $t=1, d(t, g t)<d\left(t, f g x_{n}\right)$.

Definition 2.60 (Pathak et al. [51]) Two self-mappings $f$ and $g$ of a metric space $(X, d)$ are called:

(i) $g$-biased of type $(A)$ iff

$$
\alpha \lim _{n} d\left(g x_{n}, g g x_{n}\right) \leq \alpha \lim _{n} d\left(f x_{n}, f g x_{n}\right)
$$

where $\alpha \lim _{n}$ stays for $\limsup \operatorname{sur}_{n}$ or $\liminf _{n}$, whenever $\left\{x_{n}\right\}$ is a sequence in $X$ such that $\lim _{n} f x_{n}=\lim _{n} g x_{n}=t$ for some $t$ in $X$. 
Definition 2.61 (Pathak et al. [59]) Two self-mappings $f$ and $g$ of a metric space $(X, d)$ are said to be weakly compatible mappings of type $(f)$ with index $p$ at a point $x$ in $X$ iff there exists $p>0$ such that $f x=g x$ implies

$$
d(f g x, f x)^{p}+d(f g x, g f x)^{p} \leq d(g f x, g x)^{p} .
$$

Definition 2.62 (Fisher and Murthy [53] (see also [89])) Two self-mappings $f$ and $g$ of a metric space $(X, d)$ are called biased mappings of type $\left(A_{f}\right)$ iff

$$
f x=g x \text { implies } d(g f x, f x) \leq d(f f x, g x) \text { for some } x \text { in } X \text {. }
$$

Definition 2.63 (Pathak and Tiwari [65]) Let $f$ and $g$ be mappings from a metric space $(X, d)$ into itself. The pair of mappings $f, g$ is said to be $\phi$-weakly compatible of type $(f, g)$ at $x \in X$, iff for every $p>0, f x=g x$ implies

$$
\phi\left(\frac{d(f g x, f x)^{p+1}+d(f g x, g f x)^{p+1}}{d(f g x, f x)+d(f g x, g f x)}\right) \leq d(g f x, g x)^{p},
$$

where $\phi:[0,1) \rightarrow[0,1)$ is upper semi-continuous, non-decreasing and $\phi(t)<t$ for all $t>0$, and $d(f g x, f x)+d(f g x, g f x) \neq 0$.

If $\phi(t)=h t$, where $0<h<1$, then the pair of mappings $(f, g)$ is said to be $h$-weakly compatible of type $(f, g)$.

Example 2.64 [65] Consider $X=[0,1)$ with the Euclidean metric $d$ on $X$ and $\phi(t)=\frac{1}{2} t$. Define $f, g: X \rightarrow X$ by

$$
f x=1+x \text { and } g x=1+2 x .
$$

Here for $x=0$ and $p>0,(f, g)$ is $\phi$-weakly compatible of type $(f, g)$ but $(f, g)$ is not $\phi$-weakly compatible of type $(g, f)$ for $p>1$. Moreover the pair $(f, g)$ is neither weakly commuting nor weakly compatible.

In 2008, Al-Thagafi and Shahzad [38] introduced the notion of occasionally weakly compatible (OWC) mappings as a generalization of weakly compatible mappings. While the paper [38] was under review, Jungck and Rhoades [90] used the concept of OWC and proved several results under different contractive conditions. In view of the paper of Bisht and Pant [91], under contractive conditions proving existence of common fixed points by assuming OWC as presented in [90] is equivalent to proving the existence of common fixed points by assuming the existence of common fixed points.

Moreover, it was shown by Dorić et al. in [92] that in the presence of a unique point of coincidence, the OWC condition reduces to weak compatibility. Hence, a lot of generalizations obtained by using the OWC and similar conditions are not real generalizations (see also [93-96]).

Definition 2.65 (Al-Thagafi and Shahzad [38]) Two self-mappings $f$ and $g$ of a metric space $(X, d)$ are said to be occasionally weakly compatible (OWC) if $f g x=g f x$ for some $x \in C(f, g)$. 
In [90] Jungck and Rhoades presented the following variant of OWC: Two self-mappings $f$ and $g$ of a metric space $(X, d)$ are said to be occasionally weakly compatible (in the sense of Jungck and Rhoades [90]) if there exists at least one coincidence point at which $f$ and $g$ commute, i.e., if $f x=g x$ for some $x \in X$, then $f g x=g f x$. This definition may be termed as non-trivial OWC mappings.

In [97] Al-Thagafi and Shahzad have shown that if $(X, d)$ is a discrete metric space and $C(f, g) \neq \emptyset$ then $f$ and $g$ are weakly compatible iff they are weakly commuting.

Remark 2.66 Besides commutativity of the mappings the notion of non-trivial OWC requires the mappings to have a coincidence point and, therefore, imposes a very strong condition on the mappings. By assuming the existence of a coincidence point the notion of non-trivial OWC circumvents the most crucial part of fixed point theorems consisting of constructive procedures yielding coincidence points. Conditions or constructive procedures yielding coincidence points are important parts of fixed point theorems and strong assumptions like non-trivial OWC do not and should not obviate the need for constructive procedures.

Definition 2.67 (Chen and Li [60]) Two self-mappings $f$ and $g$ of metric space $(X, d)$ are said to be Banach operator pair iff the set $F(g)$ is $f$-invariant, namely $f(F(g)) \subset F(g)$.

It is easy to check that the commuting pair $(f, g)$ is a Banach operator pair but the converse is not true in general.

Example 2.68 Let $X=[1, \infty)$ and $d$ be the usual metric on $X$. Define $f, g: X \rightarrow X$ by

$$
f x=x^{3} \text { for all } x \text { and } g x=3 x-2 \text { for all } x \text {. }
$$

Then $F(g)=\{1\}$. Here $(f, g)$ is a Banach operator pair but $f$ and $g$ are not commuting.

Definition 2.69 (Pathak and Hussain [63]) Two self-mappings $f$ and $g$ of a metric space $(X, d)$ are said to be $P$-operators iff there is a point $x \in X$ such that

$$
x \in C(f, g) \quad \text { and } \quad d(x, f x) \leq \delta(C(f, g)) \text {, }
$$

where $\delta(A)=\sup \{\max \{d(x, y), d(y, x)\}: x, y \in A\}$ for $A \subset X$.

Pathak and Hussain [63] have shown by means of an example that OWC mappings are $P$-operators. If the self-mappings $f$ and $g$ of $X$ are weakly compatible, then $g(C(f, g)) \subset$ $C(f, g)$, and hence $f$ and $g$ are $P$-operators.

Definition 2.70 (Hussain et al. [39]) Two self-mappings $f$ and $g$ of a metric space $(X, d)$ are said to be $J H$-operators iff there is a point $w=f x=g x$ in $P C(f, g)$ such that $d(w, x) \leq$ $\delta(P C(f, g))$.

Example 2.71 [63] Consider $X=[0,1)$ with the Euclidean metric $d$ on $X$. Define $f, g: X \rightarrow$ $X$ by

$$
\begin{aligned}
& f x=x^{2} \quad \text { and } \quad g x=2 x \quad \text { for all } x \neq 0, \\
& f 0=g 0=1 .
\end{aligned}
$$


Then $C(f, g)=\{0,2\}$ and $P C(f, g)=\{1,4\}$. Obviously $f$ and $g$ are $P$-operators and $J H$ operators but neither OWC mappings nor weakly compatible mappings. Further note that $F f=\{1\}$ and $g(1)=2 F(f)$, which show that $(g, f)$ is not a Banach operator pair.

Definition 2.72 (Bouhadjera and Djoudi [66], Hussain et al. [39]) Two self-mappings $f$ and $g$ of a metric space $(X, d)$ are said to be occasionally weakly $f$-biased iff there is a point $x \in X$ such that $f x=g x$ and $d(f g x, f x) \leq d(g f x, g x)$.

The definition of occasionally weakly $g$-biased can be obtained from the definition of occasionally weakly $f$-biased by interchanging the role of $f$ and $g$.

Bouhadjera and Djoudi [66] have shown that if $f$ and $g$ are OWC mappings then $f$ and $g$ are both occasionally weakly $f$-biased and $g$-biased. Hence OWC mappings is a subclass of occasionally weakly biased mappings.

It may be noted that nontrivially $(C(f, g) \neq \phi)$ weakly $f$-biased and $g$-biased mappings, respectively, are occasionally weakly $f$-biased and $g$-biased, respectively. However, the reverse implications are not true.

Example 2.73 [66] Let $X=[0, \infty)$ and $d$ be the usual metric on $X$. Define $f, g: X \rightarrow X$ as follows:

$$
\begin{aligned}
& f x=4 x^{2} \quad \text { if } 0 \leq x \leq 1, \quad f x=\frac{9}{x} \quad \text { if } 1<x<\infty, \\
& g x=1 \quad \text { if } 0 \leq x \leq 1, \quad g x=3 x \quad \text { if } 1<x<\infty .
\end{aligned}
$$

Then we have $f x=g x$ if and only if $x=1 / 2$ or $x=3$ and $d(f g(\sqrt{3}), f(\sqrt{3})) \leq d(g f(\sqrt{3})$, $g(\sqrt{3}))$, that is, the pair $\{f, g\}$ is occasionally weakly $f$-biased. But, $3=d\left(f g\left(\frac{1}{2}\right), f\left(\frac{1}{2}\right)\right) \not \leq$ $d\left(g f\left(\frac{1}{2}\right), g\left(\frac{1}{2}\right)\right)$, i.e., the pair $\{f, g\}$ is not weakly $f$-biased. Similarly we can show that $g$-occasionally weakly biased mappings may not be $g$-weakly biased mappings.

Definition 2.74 (Hussain et al. [39]) Let $d: X \times X \rightarrow[0, \infty)$ be a mapping such that $d(x, y)=0$ iff $x=y$. Two self-mappings $f$ and $g$ on $X$ are said to be $J H$-operators iff there is a point $w=f x=g x$ in $P C(f, g)$ such that $d(w, x) \leq \delta(P C(f, g))$ and $d(x, w) \leq \delta(P C(f, g))$ where $\delta(A)=\sup \{\max \{d(x, y), d(y, x)\}: x, y \in A\}$ for $A \subset X$.

Definition 2.75 (Pathak and Rai [37]) Let $d: X \times X \rightarrow[0, \infty)$ be a mapping such that $d(x, y)=0$ iff $x=y$. Two self-mappings $f$ and $g$ on $X$ are said to be $P D$-operator pair if there is a point $u \in X$ such that $u \in C(f, g)$ and $d(f g u, g f u) \leq \delta(P C(f, g))$ where $\delta(A)=$ $\sup \{\max \{d(x, y), d(y, x)\}: x, y \in A\}$ for $A \subset X$.

Example 2.76 [37] Let $X=[0,1]$ and let $d=|x-y|^{2}$ be the metric. Define $f, g: X \rightarrow X$ by $f x=x^{2}$ and $g x=\frac{x}{2}$, for all $x \neq 0$ and $f 0=g 0=1$. Since $C(f, g)=\{0,1 / 2\}$ and $P C(f, g)=$ $\{1,1 / 4\}, \operatorname{diam}(C(f, g))=1 / 4$ and $\operatorname{diam}(P C(f, g))=\frac{9}{16}$. Clearly, $(f, g)$ is $P D$-operator pair, but not commuting, not weakly compatible and not OWC.

Definition 2.77 (Pant and Pant [61]) Two self-mappings $f$ and $g$ of a metric space $(X, d)$ are called conditionally commuting if they commute on a nonempty subset of the set of coincidence points whenever the set of their coincidences is nonempty. 
Example 2.78 [61] Let $X=[0,1]$ and $d$ be the usual metric on $X$. Define self-mappings $f$ and $g$ on $X$ as follows:

$$
\begin{aligned}
& f(x)=\left(\frac{\sqrt{5-4(2 x-1)^{2}}-1}{4}\right), \\
& g(x)=\frac{1-x}{3} .
\end{aligned}
$$

Then $C(f, g)=\left\{1, \frac{1}{4}\right\}$ and $f g 1 \neq g f 1$. Hence $f$ and $g$ are conditionally commuting but not weakly compatible. Further $f$ and $g$ are noncompatible mappings. To see this let us consider a sequence $\left\{x_{n}\right\}$ given by $x_{n}=1-\frac{1}{n}$. Then $f_{x_{n}} \rightarrow 0, g x_{n} \rightarrow 0, f g x_{n} \rightarrow 0, g f x_{n} \rightarrow \frac{1}{3}$ and $\lim _{n} d\left(f g x_{n}, g f x_{n}\right) \neq 0$.

Remark 2.79 In this remark we highlight the difference (in terms of applicability) between the three concepts: weak compatibility, occasionally weak compatibility and conditionally commutativity. A generalized Lipschitz type pair $(f, g)$ of self-mappings of a metric space $(X, d)$ may exhibit any of the conditions: (i) $f$ and $g$ do not possess a coincidence point, (ii) $f$ and $g$ possess coincidence points and commute at each coincidence point, and (iii) $f$ and $g$ possess more than one coincidence points and commute on a proper subset of the set of their coincidence points. The notions of commuting, weak commuting, compatible, pointwise $R$-weak commuting, or weak compatible mappings apply in conditions (i) and (ii) but do not apply in condition (iii). The notion of non-trivial OWC mappings applies in conditions (ii) and (iii) but does not apply in condition (i) as it presupposes the existence of a coincidence point. The notions of OWC and conditional commutativity are applicable in each of the three conditions mentioned above.

In 2009, Bouhadjera and Godet-Thobie [62] introduced the notion of subcompatibile mappings.

Definition 2.80 (Bouhadjera and Godet-Thobie [62]) Two self-mappings $f$ and $g$ of a metric space $(X, d)$ are called subcompatible iff there exists a sequence $\left\{x_{n}\right\}$ such that $\lim _{n \rightarrow \infty} f x_{n}=\lim _{n \rightarrow \infty} g x_{n}=t$ and $\lim _{n \rightarrow \infty} d\left(f g x_{n}, g f x_{n}\right)=0$.

Example 2.81 [62] Let $X=[0, \infty)$ and $d$ be the usual metric on $X$. Define $f, g: X \rightarrow X$ as follows:

$$
\begin{aligned}
& f x=x^{2} \quad \text { for all } x \in X, \\
& g x=x+2 \quad \text { if } 0 \leq x \leq 4 \text { and } 9<x<\infty, \quad g x=x+12 \quad \text { if } 4<x \leq 9 .
\end{aligned}
$$

Then $f$ and $g$ are subcompatible but not OWC. To see this consider the sequence $\left\{x_{n}\right\}$ given by $x_{n}=2+\frac{1}{n}$. Then $f x_{n} \rightarrow 4, g x_{n} \rightarrow 4, f g x_{n} \rightarrow 16, g f x_{n} \rightarrow 16$ and $\lim _{n} d\left(f g x_{n}, g f x_{n}\right)=0$. On the other hand, we have $f x=g x$ iff $x=2$ and $f g 2 \neq g f 2$, hence mappings $f$ and $g$ are not OWC.

Remark 2.82 Above Example 2.81 shows that subcompatible mappings need not imply commutativity at the coincidence point. It may be observed that subcompatible mappings are independent from the compatible mappings and in the setting of a unique common 
fixed point (or unique point of coincidence), subcompatibility does not reduce to the class of compatibility. The following examples illustrate these facts.

Example 2.83 Let $X=[0, \infty)$ and $d$ be the usual metric on $X$. Define $f, g: X \rightarrow X$ by

$$
f x=x \text { for all } x \text { and } g x=2 x \text { for all } x \text {. }
$$

Then $f$ and $g$ are compatible but not subcompatible.

Example 2.84 Let $X=[1,8]$ and $d$ be the usual metric on $X$. Define $f, g: X \rightarrow X$ as follows:

$$
\begin{aligned}
& f x=2 \quad \text { if } x \leq 2, \quad f x=6 \quad \text { if } x>2, \\
& g x=6-2 x \quad \text { if } x \leq 2, \quad g x=8 \quad \text { if } x>2 .
\end{aligned}
$$

It may be observed that $f$ and $g$ are subcompatible mappings. To see that $f$ and $g$ are subcompatible, let us consider the constant sequence $\left\{x_{n}\right\}$ given by $x_{n}=2$. Then $f x_{n} \rightarrow 2$, $g x_{n} \rightarrow 2, f g x_{n} \rightarrow 2, g f x_{n} \rightarrow 2$, and $\lim _{n} d\left(f g x_{n}, g f x_{n}\right)=0$. Further, if we consider $\left\{y_{n}\right\}$ given by $y_{n}=2-\frac{1}{n}: n>0$, then $f y_{n} \rightarrow 2, g y_{n} \rightarrow 2$, and $\lim _{n} d\left(f g y_{n}, g f y_{n}\right) \neq 0$. Thus $f$ and $g$ are subcompatible but not compatible.

Remark 2.85 The notion of subcompatibility imposes a strong condition on the mappings $f$ and $g$ by requiring the existence of a sequence $\left\{x_{n}\right\}$ such that $f x_{n} \rightarrow t, g x_{n} \rightarrow t$. Such a precondition is not required in order that $f$ and $g$ be compatible. In [40] Pant and Bisht introduced the notion of conditional compatibility which does not require such a precondition and yet is a proper generalization of both nontrivial compatibility and subcompatibility.

Definition 2.86 (Pant and Bisht [40]) Two self-mappings $f$ and $g$ of a metric space $(X, d)$ are called conditionally compatible if and only if whenever the set of sequences $\left\{x_{n}\right\}$ satisfying $\lim _{n \rightarrow \infty} f x_{n}=\lim _{n \rightarrow \infty} g x_{n}$ is nonempty, there exists a sequence $\left\{y_{n}\right\}$ such that $\lim _{n \rightarrow \infty} f y_{n}=\lim _{n \rightarrow \infty} g y_{n}=t$ (say) and $\lim _{n \rightarrow \infty} d\left(f g y_{n}, g f y_{n}\right)=0$.

In [67], Pant and Bisht introduced a new notion of pseudo compatible mappings, which is a stronger version of conditionally compatible mappings.

Let $f, g$ be self-mappings of a metric space $(X, d)$. Then for a sequence $\left\{y_{n}\right\}$ in $X$ satisfying $\lim _{n \rightarrow \infty} f x_{n}=\lim _{n \rightarrow \infty} g y_{n}$, a sequence $\left\{z_{n}\right\}$ is called an associated sequence if $f y_{n}=g z_{n}$ or $g y_{n}=f z_{n}$ and $\lim _{n \rightarrow \infty} f z_{n}=\lim _{n \rightarrow \infty} g z_{n}$.

Definition 2.87 (Pant and Bisht [67]) Two self-mappings $f$ and $g$ of a metric space $(X, d)$ are called pseudo compatible iff whenever the set of sequences $\left\{x_{n}\right\}$ satisfying $\lim _{n \rightarrow \infty} f x_{n}=$ $\lim _{n \rightarrow \infty} g x_{n}$ is nonempty, there exists a sequence $\left\{y_{n}\right\}$ such that $\lim _{n \rightarrow \infty} f y_{n}=\lim _{n \rightarrow \infty} g y_{n}=$ $t$ (say), $\lim _{n \rightarrow \infty} d\left(f g y_{n}, g f y_{n}\right)=0$ and $\lim _{n \rightarrow \infty} d\left(f g z_{n}, g f z_{n}\right)=0$ for any associated sequence $\left\{z_{n}\right\}$ of $\left\{y_{n}\right\}$.

Suzuki and Pathak [64] also extended the class of compatible type mappings and several analogous notions to almost compatible mappings and analogous notions, respectively. 
Definition 2.88 Two self-mappings $f$ and $g$ of a metric space $(X, d)$ are called to be almost compatible iff $f$ and $g$ are compatible and the following hold for every sequence $\left\{x_{n}\right\}$ in $X$ :

(C1) $f x_{n+1}=g x_{n}$ for $n \in \mathbb{N}$.

(C2) $\left\{x_{n}\right\}$ converges.

(C3) $\left\{g f x_{n}\right\}$ is bounded.

In 2013, Bisht and Shahzad [41] redefined the notion of conditionally compatible mappings by faintly compatible mappings.

Definition 2.89 (Bisht and Shahzad [41]) Two self-mappings $f$ and $g$ of a metric space $(X, d)$ are called to be faintly compatible iff $f$ and $g$ are conditionally compatible and $f$ and $g$ commute at the subset of coincidence points, whenever the set of coincidence points is nonempty.

Example 2.90 Let $X=[0,1]$ and $d$ be the usual metric on $X$. Define self-mappings $f$ and $g$ on $X$ as follows:

$$
\begin{aligned}
& f(x)=\frac{1}{2}-\left|\frac{1}{2}-x\right|, \\
& g(x)=\frac{2}{3}(1-x) .
\end{aligned}
$$

Then $f$ and $g$ are faintly compatible but neither compatible nor weakly compatible.

Remark 2.91 In recent works several authors claimed to introduce some weaker noncommuting notions and pretended to show, weak compatibility as a proper subclass of their weaker notions. This is, however, not true. In view of the results of Dorić et al. [92] (see also Alghamdi et al. [94], Kadelburg et al. [96], Pant and Bisht [40] and Bisht and Rakocević [95]) most of the generalized commutativity notions fall in the subclass of weak compatibility in the setting of a unique common fixed point (or unique point of coincidence).

The following lemma was presented by Abbas and Jungck [98].

Lemma 2.92 [98] If a weakly compatible (WC) pair $(f, g)$ of self-mappings on $X$ has a unique POC, then it has a unique common fixed point.

Jungck and Rhoades [90] established the following lemma.

Lemma 2.93 [90] If an OWC pair $(f, g)$ of self-mappings on $X$ has a unique POC, then it has a unique common fixed point.

The following result is due to Dorić et al. [92].

Proposition 2.94 [92] Let a pair of mappings $(f, g)$ have a unique POC. Then it is WC if and only if it is $O W C$.

Proposition 2.95 [94] Let $d: X \times X \rightarrow[0, \infty)$ be a mapping such that $d(x, y)=0$ if and only if $x=y$. Let a pair of mappings $(f, g)$ have a unique POC. If it is a pair of JH-operators, then it is $W C$. 
Proposition 2.96 [94] Let d be symmetric on X. Let a pair of mappings $(f, g)$ have a unique $P O C$ which belongs to $F(f)$. If it is a pair of occasionally weakly g-biased mappings, then it is $W C$.

Proposition 2.97 [94] Let d be symmetric on $X$, and let a pair of mappings $(f, g)$ have a unique $C P$, that is, $C(f, g)$ is a singleton. If $(f, g)$ is P-operator pair, then it is $W C$.

Proposition 2.98 [94] Let d be symmetric on $X$, and let a pair of mappings $(f, g)$ have a unique POC. Then it is weakly g-biased if and only if it is occasionally weakly g-biased.

Proposition 2.99 [95] Let $d: X \times X \rightarrow[0, \infty)$ be a mapping such that $d(x, y)=0$ if and only if $x=y$. Let a pair of mappings $(f, g)$ have a unique POC. If it is a pair of PD-operators, then it is WC.

Let $\phi: R_{+} \rightarrow R_{+}$be a non-decreasing function satisfying the condition $\phi(t)<t$, for each $t>0$.

Proposition 2.100 [95] Let $d: X \times X \rightarrow[0, \infty)$ be a mapping such that $d(x, y)=0$ if and only if $x=y$. Suppose $(f, g)$ is PD-operator pair and satisfy the condition:

$$
d(f x, f y) \leq \phi(\max \{d(g x, g y), d(g x, f y), d(f x, g y), d(g y, f y)\})
$$

for each $x, y \in X$. Then $f$ and $g$ are $W C$.

Theorem 2.101 [95] Under the contractive condition (2.1) assumed in the above proposition, the assumption of PD-operators and the existence of a unique common fixed point are equivalent conditions.

More recently, Kadelburg et al. [96] have shown that some of noncommuting conditions which are formally distinct from each other, actually coincide in the case when the given mappings have a unique point of coincidence. Hence, many new common fixed point results cannot be incurred in this way. The following proposition was proved in [96].

Proposition 2.102 Let $(X, d)$ be a metric space, and let $f, g: X \rightarrow X$. Let the pair $(f, g)$ have exactly one point of coincidence. Then the noncommuting conditions weakly compatible, occasionally weakly compatible, conditionally commuting and PD-operators are equivalent, and equivalent with the condition that the pair $(f, g)$ has a unique common fixed point.

Remark 2.103 It is important to note that majority of the noncommuting conditions before weak compatibility do not presume the existence of a coincidence point but actually establish the existence of a coincidence point by using these conditions. In fact, it can easily be established that some of these conditions, e.g., weakly commuting etc. not only imply commutativity at coincidence points but in combination with other sufficient conditions also imply the existence of the coincidence point. Thus we can say that with the inception of the definition of non-trivial OWC mappings more focus would be given to those noncommuting conditions which directly assume the existence of coincidence point, which is relatively strong condition in comparison to weakly compatible mappings. 
Remark 2.104 If there are just two mappings involved, and they only have one coincidence point (which turns out to be the unique fixed point), then, of course, most of the generalizations of commutativity coincide with weak compatibility. Recently, Pant and Bisht [40] (see also [91, 95]) have shown that under contractive conditions proving existence of common fixed points by assuming several weaker noncommuting notions is equivalent to proving the existence of common fixed points by assuming the existence of common fixed points. Hence such type of results are merely redundant exercises.

\section{Applications}

Fixed point theory has played central role in the problems of nonlinear analysis. Common fixed point theorems have provided powerful tools in demonstrating the existence solutions to large variety of problem in applied mathematics. In this section we give some of the areas where common fixed point theorems are applicable.

\subsection{Applications in differential equations}

In [5] Goebel gave a nice application of the coincidence theorem (Theorem 1.1) for the solution of differential equation. He considered the following differential equation:

$$
\dot{x}=s(t, x)
$$

(cf. [99]). Suppose that the function $\{s(t, x)\}$ is defined in the half plane $t \geq 0,-\infty<x<+\infty$ and satisfies Caratheodory's conditions (cf. [100]) and Lipschitz inequality

$$
|s(t, x)-s(t, y)| \leq L(t)|x-y|
$$

where $\{L(t)\}$ is locally integrable on the interval $\langle 0, \infty)$.

$$
\text { If } \int_{0}^{t} s(\tau, 0) d \tau=O\left(\exp \int_{0}^{t} L(\tau) d \tau\right) \text { and } p>1 \text { then the transformations } f \text { and } g \text { defined by }
$$

$$
\begin{aligned}
& (f x)(t)=\left\{\int_{0}^{t} s(\tau, x(\tau)) d \tau+\xi\right\} \exp \left\{-p \int_{0}^{t} L(\tau) d \tau\right\}, \\
& (g x)(t)=x(t) \exp \left\{-p \int_{0}^{t} L(\tau) d \tau\right\}
\end{aligned}
$$

mappings the set $A=\left[\{x(t)\}: x(t)=O\left(\exp \int_{0}^{t} L(\tau) d \tau\right)\right]$ into the Banach space $B$ of continuous functions on $\langle 0, \infty)$ with the norm $|x|=\sup _{\langle 0, \infty)}|x(t)|$, then Theorem 1.1 assures that there exists a function $\{\bar{x}(t)\} \in A$ which satisfies $f \bar{x}=g \bar{x}$ and $\bar{x}(t)=\int_{0}^{t} s(\tau, \bar{x}(\tau)) d \tau+\xi$ is the unique solution in the sense of Caratheodory [100] with the initial condition $\bar{x}(0)=\xi$ for every $\xi$.

\subsection{Applications in approximation theory}

Fixed point theorems have been extensively used in the existence and uniqueness of invariant approximations. In 1963, Meinardus [101] utilized the Schauder fixed point theorem [102] to prove the very first result regarding invariant approximation. Afterwards, Brosowski [103] and Subrahmanyam [104] generalized the result of Meinardus under different settings. Further significant contribution in this area was made by a number of authors. They all considered one mapping or a pair of commuting mappings to show the 
existence of invariant approximation. In 1998, the study of invariant approximations for noncommuting mappings was initiated by Shahzad [105] (see also [106, 107]). Since then this area has been flourished further and many interesting results appeared for noncommuting mappings.

Let $D$ be a nonempty subset of a normed space $X, f$ and $g$ self-mappings of $D$, and $F(f)$ (respectively $F(g)$ ) the set of fixed points of $f$ (respectively $g$ ). The self-mapping $g$ is called:

(i) $f$-contraction if $\|g x-g y\| \leq k\|f x-f y\|$ for all $x, y \in D$ and some $k \in[0,1)$;

(ii) $f$-nonexpansive if $\|g x-g y\| \leq\|f x-f y\|$ for all $x, y \in D$;

(iii) nonexpansive if $\|g x-g y\| \leq\|x-y\|$ for all $x, y \in D$.

For $p \in X$, let $\delta(p, D):=\inf _{z \in D}\|z-p\|$. The set $D$ is called:

(iv) $q$-starshaped if $k x+(1-k) q \in D$ for all $x \in D$ and all $k \in[0,1]$;

(v) convex if $k x+(1-k) y \in D$ for all $x, y \in D$ and all $k \in[0,1]$.

The self-mapping $f$ is called:

(vi) affine if $D$ is convex and $f(k x+(1-k) y)=k f x+(1-k) f y$ for all $x, y \in D$ and all $k \in[0,1]$;

(vii) $q$-affine if $D$ is $q$-starshaped and $f(k x+(1-k) q)=k f x+(1-k) q$ for all $x \in D$ and all $k \in[0,1]$.

Note that $f q=q$ whenever $f$ is a $q$-affine self-mapping of a $q$-starshaped set $D$.

(viii) $R$-subweakly commuting (w.r.t. $q$ ) [107] if $D$ is $q$-starshaped with $q \in F(f)$ and $\|g f x-f g x\| \leq R \delta(f x,[g x, q])$ for all $x \in D$ and some $R>0$;

(ix) $R$-subcommuting (w.r.t. $q$ ) $[106,108]$ if $D$ is $q$-starshaped with $q \in F(f)$ and $\|g f x-f g x\| \leq \frac{R}{k}\|k g x+(1-k) q-f x\|$ for all $x \in D, k \in(0,1]$ and some $R>0$.

In fact, $R$-subcommutativity (w.r.t. $q)$ is equivalent to $\|g f x-f g x\| \leq R \delta(f x,[g x, q))$ for all $x \in D$ and some $R>0$. Hence, $R$-subweak commutativity implies $R$-subcommutativity. However, $R$-subweak commutativity is implied by $R$-subcommutativity whenever $\delta(f x,[g x$, $q))=\delta(f x,[g x, q])($ for more details see [108]).

Let $D$ be a $q$-starshaped subset of a normed space $X, f$ and $g$ self-maps of $D$ with $q \in F(f)$, and $C_{q}(f, g):=\bigcup\left\{C\left(f, g_{k}\right): 0 \leq k \leq 1\right\}$ where $g_{k} x:=k g x+(1-k) q$. The self-mappings $f$ and $g$ are called $C_{q}$-commuting [108] if $f g x=g f x$ for all $x \in C_{q}(f, g)$. Clearly, $C_{q}$-commuting selfmappings are weakly compatible. In general, the converse does not hold as the following example shows.

Example 3.1 [108] Let $X=\mathbb{R}$ with the usual norm and $D=[0, \infty)$. Define $f, g: D \rightarrow D$ by

$$
f(x)=2 x \quad \text { and } \quad g(x)= \begin{cases}x^{2} & \text { if } x \neq 2 \\ 1 & \text { if } x=2\end{cases}
$$

Then $D$ is $q$-starshaped with $q=0, C(f, g)=\{0\}$ and $C_{q}(f, g)=\{0\} \cup[2, \infty)$. Moreover, $f$ and $g$ are weakly compatible but not $C_{q}$-commuting.

Note that $R$-subweakly commuting and $R$-subcommuting self-mappings are $C_{q}$-commuting, but the converse does not hold in general. The following two examples show that $C_{q}$-commuting and $R$-weakly commuting self-mappings are different classes.

Example 3.2 [108] Let $X=\mathbb{R}$ with the usual norm and $D=[0, \infty)$. Define $f, g: D \rightarrow D$ by

$$
\begin{array}{ll}
f x=x / 2 & \text { if } 0 \leq x<1, \quad f x=x \quad \text { if } x \geq 1, \\
g x=1 / 2 & \text { if } 0 \leq x<1, \quad g x=x^{2} \quad \text { if } x \geq 1 .
\end{array}
$$


Then $D$ is $q$-starshaped with $q=1$ and $C_{q}(f, g)=[1, \infty)$. Moreover, $f$ and $g$ are $C_{q}$-commuting but not $R$-weakly commuting for all $R>0$. Hence, $f$ and $g$ are neither $R$-subweakly commuting nor $R$-subcommuting.

Example 3.3 [108] Let $X=\mathbb{R}$ with the usual norm and $D=[1, \infty)$. Define $f, g: D \rightarrow D$ by

$$
f x=2 x-1 \quad \text { and } \quad g x=x^{2} \quad \text { for all } x \in D
$$

Then $D$ is $q$-starshaped with $q=1$ and $C_{q}(f, g)=[1, \infty)$. Moreover, $f$ and $g$ are $R$-weakly commuting with $R=2$ (see [36]) but not $C_{q}$-commuting.

For a nonempty subset $M$ of $X$ and $p \in X$, let $B_{M}(p):=\{x \in M:\|x-p\|=\delta(p, M)\}$, $C_{M}^{I}(p):=\left\{x \in M: I x \in B_{M}(p)\right\}$ and $M_{p}:=\{x \in M:\|x\| \leq 2\|p\|\}$. The set $B_{M}(p)$ is called the set of best $M$-approximants to $p$. Let $\mathcal{C}_{0}$ (respectively $\mathcal{C}_{0}^{w}$ ) denote the class of closed (respectively weakly closed) convex subsets of $X$ containing 0 . Note that $B_{M}(p) \subseteq M_{p} \in \mathcal{C}_{0}$ whenever $M \in \mathcal{C}_{0}$. A self-mapping $g: X \rightarrow X$ is said to be compact on $M$ if whenever $A$ is a nonempty bounded subset of $M$, then $\overline{g(A)}$ is compact. A mapping $h: D \rightarrow X$ is said to be demiclosed at $y \in X$ if whenever $\left\{x_{n}\right\}$ is a sequence in $D$ such that $x_{n} \rightarrow z \in D$ weakly and $h x_{n} \rightarrow y$ strongly, then $h z=y$. A Banach space $X$ is said to satisfy Opial's condition if whenever $\left\{x_{n}\right\}$ is a sequence in $X$ such that $x_{n} \rightarrow z \in X$ weakly, then

$$
\liminf _{n \rightarrow \infty}\left\|x_{n}-z\right\|<\liminf _{n \rightarrow \infty}\left\|x_{n}-x\right\|
$$

holds for all $x \neq z$. Note that Hilbert and $l^{p}(1<p<\infty)$ spaces satisfy Opial's condition.

In 1969, Brosowski [103] proved the following result.

Theorem 3.4 Let $f$ be a contractive linear operator on a normed linear space X. Let $M$ be an $f$-invariant subset of $X$ and $p$ an $f$-invariant point. If the set of best $M$-approximants to $p$, i.e., $B_{M}(p)$ is nonempty, compact, and convex, then it contains an $f$-invariant point.

In [104] Subrahmanyam superseded the necessity of nonemptyness of $B_{M}(p)$ by the assumption that $M$ is a finite-dimensional subspace of $X$.

Theorem 3.5 (Subrahmanyam [104]) Let $X$ be a normed space, $f$ a nonexpansive selfmapping of $X, M$ a nonempty subset of $X, f(M) \subseteq M$, and $p \in F(f)$. Then $F(f) \cap B_{M}(p)$ is nonempty provided $M$ is a finite-dimensional subspace of $X$.

In 1979, Singh [109] demonstrated that the Brosowski [103] result remains true if linearity of the operator $f$ is dropped and convexity is replaced by starshaped property. Singh's [110] result remains true when contractive nature of $f$ is replaced by nonexpansiveness of $f$ on $B_{M}(p) \cup\{p\}$.

Theorem 3.6 (Singh [110]) Letf be a contractive operator on a normed linear space X. Let $f(M) \subseteq M$ and $p \in F(f)$. If $B_{M}(p)$ is nonempty, compact, and starshaped, then it contains anf-invariant point.

Hicks and Humphries [111] observed that Theorem 5.6 of Singh is also true if the assumption $f(M) \subseteq M$ is replaced by $f(\partial M) \subseteq M$, where $\partial M$ denotes the boundary of $M$. 
Theorem 3.7 (Hicks and Humphries [111]) Let $f$ be a contractive operator on a normed linear space $X$. Let $f(\partial M) \subseteq M$ and $p \in F(f)$. If $B_{M}(p)$ is nonempty, compact, and starshaped, then it contains an $f$-invariant point.

Smoluk [112] observed that the finite-dimensionality of $M$ in Subrahmanyam's result can be replaced by the assumptions that $f$ is linear and that $\overline{f(D)}$ is compact for every bounded subset $D$ of $M$.

Theorem 3.8 (Smoluk [112]) Let $X$ be a normed space, $f$ a nonexpansive self-mapping of $X, M$ a nonempty subset of $X, f(M) \subseteq M$, and $p \in F(f)$. Then $F(f) \cap B_{M}(p)$ is nonempty provided $f$ is linear, $M$ is a closed subspace of $X$, and $f$ is compact on $M$.

Habiniak [113] dropped the linearity of $f$ from Smoluk's result (see also [114]).

Theorem 3.9 (Habiniak [113]) Let $X$ be a normed space, $f$ a nonexpansive self-mapping of $X, M$ a nonempty subset of $X, f(M) \subseteq M$, and $p \in F(f)$. Then $F(f) \cap B_{M}(p)$ is nonempty provided $M$ is a closed subspace of $X$ and $f$ is compact on $M$.

Following $[108,115]$, we summarize above invariant approximation results as follows.

Theorem 3.10 Let $X$ be a normed space, $f$ a nonexpansive self-mapping of $X, M a$ nonempty subset of $X$ and $p \in F(f)$. Then $F(f) \cap B_{M}(p)$ is nonempty provided one of the following conditions is satisfied:

(a) $f$ is linear, $f(M) \subseteq M$ and $B_{M}(p)$ is nonempty, compact, and convex (Brosowski [103]),

(b) $f(M) \subseteq M$ and $M$ is a finite-dimensional subspace of $X$ (Subrahmanyam [104]),

(c) $f(M) \subseteq M, B_{M}(p)$ is compact and q-starshaped (Singh [110]),

(d) $f(\partial M) \subseteq M, B_{M}(p)$ is compact and q-starshaped (Hicks and Humphries [111]),

(e) $f(M) \subseteq M$, $f$ is linear, $M$ is a closed subspace of $X$, and $f$ is compact on $M$ (Smoluk [112]),

(f) $f(M) \subseteq M, M$ is a closed subspace of $X$ and $f$ is compact on $M$ (Habiniak [113]).

In 1988, Sahab et al. [116] initiated the study of approximation results for a pair of selfmappings and extended the results of Hicks and Humphries [111] and Singh [110] to commuting mappings.

Theorem 3.11 (Sahab et al. [116], see also O'Regan and Shahzad [115]) Let $f$ and $g$ be selfmappings of a normed space $X, M \subset X$ such that $f(\partial M) \subset M$, and $p \in F(f, g)$. Suppose $f$ is $g$-nonexpansive on $B_{M}(p) \cup\{p\}, g$ is linear and continuous on $B_{M}(p)$, and $f$ and $g$ are commuting on $B_{M}(p)$. If $B_{M}(p)$ is nonempty, compact, and q-starshaped with $q \in F(g)$, and if $g\left(B_{M}(p)\right)=B_{M}(p)$, then $B_{M}(p) \cap F(f, g)$ is nonempty.

In 1995, Jungck and Sessa [117] proved the following theorem in best approximation theory, which improves and extends several theorems summarized in Theorem 3.10.

Theorem 3.12 (Jungck and Sessa [117]) Let $f$ and $g$ be self-mappings of a Banach space $X$, $M \subset X$ such that $f(\partial M) \subset M$, and $p \in F(f, g)$. Suppose $f$ is $g$-nonexpansive on $B_{M}(p) \cup\{p\}$, 
$g$ is affine and continuous in the weak topology on $B_{M}(p)$, and $f$ and $g$ are commuting on $B_{M}(p)$. If $B_{M}(p)$ is nonempty, $q$-starshaped with $q \in F(g)$, and $g\left(B_{M}(p)\right)=B_{M}(p)$, then $B_{M}(p) \cap F(f, g)$ is nonempty, provided either $(\mathrm{i}) B_{M}(p)$ is weakly compact, and $g-f$ is demiclosed; or (ii) $B_{M}(p)$ is weakly compact and $X$ satisfies Opial's condition.

The following results of Al-Thagafi and Shahzad [108] extend parts (a), (b), (c), and (d) of Theorem 3.10, results of Sahab et al. [116], Singh [109], and Jungck and Sessa [117].

Theorem 3.13 (Al-Thagafi and Shahzad [108]) Let $X$ be a normed space, $f$ and $g$ self-maps of $X$ with $p \in F(f, g)$, and $M \subseteq X$ with $f(\partial M \cap M) \subseteq M$. Suppose that $B_{M}(p)$ is closed and $q$-starshaped, $f$ and $g$ are $C_{q}$-commuting on $B_{M}(p), g\left(B_{M}(p)\right)=B_{M}(p), g$ is q-affine on $B_{M}(p)$, $f$ is continuous on $B_{M}(p)$ and $g$-nonexpansive on $B_{M}(p) \cup\{p\}$, and $\overline{f\left(B_{M}(p)\right)}$ is compact. Then $B_{M}(p) \cap F(f, g)$ is nonempty.

Theorem 3.14 (Al-Thagafi and Shahzad [108]) Let $X$ be a Banach space, $f$ and $g$ selfmaps of $X$ with $p \in F(f, g)$, and $M \subseteq X$ with $f(\partial M \cap M) \subseteq M$. Suppose that $B_{M}(p)$ is weakly closed and $q$-starshaped, $g\left(B_{M}(p)\right)=B_{M}(p), g$ is q-affine and weakly continuous on $B_{M}(p)$, $f$ and $g$ are $C_{q}$-commuting on $B_{M}(p), f$ is g-nonexpansive on $B_{M}(p) \cup\{p\},{\overline{f\left(B_{M}(p)\right)}}^{w}$ is weakly compact, and either $g-f$ is demiclosed at zero, or $X$ satisfies Opial's condition. Then $B_{M}(p) \cap F(f, g)$ is nonempty.

The following results of Al-Thagafi and Shahzad extend parts (e) and (f) of Theorem 3.10, results of Al-Thagafi [118] and Shahzad [107].

Theorem 3.15 (Al-Thagafi and Shahzad [108]) Let $X$ be a normed space, $f$ and $g$ selfmaps of $X$ with $p \in F(f, g)$, and $M \in \mathcal{C}_{0}$ with $f\left(M_{p}\right) \subseteq g(M) \subseteq M$. Suppose that $\|g x-p\|=\| x-$ $p \|$ for all $x \in M, f$ is $g$-nonexpansive on $M_{p} \cup\{p\}$, and $\overline{g\left(M_{p}\right)}$ is compact. Then $B_{M}(p)$ is nonempty, closed, and convex and $f\left(B_{M}(p)\right) \subseteq g\left(B_{M}(p)\right) \subseteq B_{M}(p)$. If, in addition, $g$ is nonexpansive on $B_{M}(p)$, then $B_{M}(p) \cap F(g)$ and $B_{M}(p) \cap F(f)$ are nonempty. If, for some $q \in B_{M}(p), g$ is q-affine, $f$ and $g$ are $C_{q}$-commuting on $B_{M}(p)$, and $\overline{f\left(B_{M}(p)\right)} \subseteq g\left(B_{M}(p)\right)$, then $B_{M}(p) \cap F(f, g)$ is nonempty.

Theorem 3.16 (Al-Thagafi and Shahzad [108]) Let $X$ be a normed space, $f$ and $g$ selfmaps of $X$ with $p \in F(f, g)$, and $M \in \mathcal{C}_{0}$ with $f\left(M_{p}\right) \subseteq g(M) \subseteq M$. Suppose that $\|g x-p\|=\|x-p\|$ for all $x \in M, f$ is g-nonexpansive on $M_{p} \cup\{p\}$, and $\overline{f\left(M_{p}\right)}$ is compact. Then $B_{M}(p)$ is nonempty, closed, and convex and $f\left(B_{M}(p)\right) \subseteq g\left(B_{M}(p)\right) \subseteq B_{M}(p)$. If, in addition, $f$ is nonexpansive on $B_{M}(p)$, then $B_{M}(p) \cap F(f)$ is nonempty. If, for some $q \in B_{M}(p), g$ is $q$-affine, $f$ and gare $C_{q}$-commuting on $B_{M}(p)$, and $\overline{f\left(B_{M}(p)\right)} \subseteq g\left(B_{M}(p)\right)$, then $B_{M}(p) \cap F(f, g)$ is nonempty.

Several other approximation results including invariant approximations results for starshaped sets and convex sets can be found in [38, 60, 105-108, 115, 119-129].

Some applications of the fixed point theorems to best simultaneous approximation are given in [130-136]. Some other applications of approximation theory can be found in [119, $121,132,137-140]$.

\subsection{Several other applications}

Several authors, have used common fixed point techniques, to obtain the existence and uniqueness of common solutions for certain class of the functional equations arise in dy- 
namic programming $[37,47,73,82,141]$. The solutions of variational inequalities arise in the two point obstacle problem, and they have been studied by several authors as an application of several common fixed point theorems [37, 48, 59, 141]. Common fixed point theorems have also been utilized to find solutions of nonlinear integral equations $[88,142$, 143] and continuous solutions for nonlinear integral inclusions [144].

\section{Competing interests}

The authors declare that they have no competing interests.

Authors' contributions

All authors read and approved the final manuscript.

\section{Author details}

${ }^{1}$ Department of Mathematics, Texas A\&M University-Kingsville, 700 University Blvd., Kingsville, TX 78363-8202, USA. ${ }^{2}$ Department of Mathematics, King Abdulaziz University, P.O. Box 80203, Jeddah, 21589, Saudi Arabia. ${ }^{3}$ Department of Mathematics - Applied Sciences and Humanities, Bipin Tripahti Kumaon Institute of Technology, Almora, Dwarahat, Uttarakhand 262553, India.

\section{Acknowledgements}

The authors are thankful to the referees for their valuable suggestions for improving the presentation of the paper. This article was funded by the Deanship of Scientific Research (DSR), King Abdulaziz University, Jeddah. The third author acknowledges with thanks DSR for financial support.

Received: 16 September 2013 Accepted: 14 January 2014 Published: 13 Feb 2014

\section{References}

1. Isbell, JR: Commuting mappings of tree. Bull. Am. Math. Soc. 10, 419 (1957)

2. Joshi, MC, Bose, RK: Some Topics in Nonlinear Functional Analysis. Wiley Eastern, New Delhi (1985)

3. Boyce, WB: Commuting functions with common fixed point. Trans. Am. Math. Soc. 137, 77-92 (1969)

4. Huneke, JP: On common fixed points of commuting continuous functions on an interval. Trans. Am. Math. Soc. 139 371-381 (1969)

5. Goebel, K: A coincidence theorem. Bull. Acad. Pol. Sci., Sér. Sci. Math. Astron. Phys. 16, 733-735 (1968)

6. Machuca, R: A coincidence theorem. Am. Math. Mon. 74, 569-572 (1967)

7. Singh, SL, Tomar, A: Weaker forms of commuting maps and existence of fixed points. J. Korea Soc. Math. Educ. Ser. B Pure Appl. Math. 10(3), 145-160 (2003)

8. Pant, RP, Bisht, RK: Common fixed point theorems under a new continuity condition. Ann. Univ. Ferrara 58, 127-141 (2012)

9. Pant, RP: A Study on Fixed Points of Noncommuting Mappings. DSc thesis, Kumaun University, Nainital, India (2000)

10. Jungck, G: Commuting mappings and fixed points. Am. Math. Mon. 83(4), 261-263 (1976)

11. Chang, SS: A common fixed point theorem for commuting mappings. Proc. Am. Math. Soc. 83(3), 645-652 (1981)

12. Das, KM, Naik, KV: Common fixed point theorems for commuting maps on metric spaces. Proc. Am. Math. Soc. 77(3), 369-373 (1979)

13. Fisher, B: Mappings with a common fixed point. Math. Semin. Notes, Kobe Univ. 7(1), 81-84 (1979)

14. Fisher, B, Sessa, B: Two common fixed point theorems for weakly commuting mappings. Period. Math. Hung. 20(3), 207-218 (1989)

15. Jungck, G: Periodic and fixed points, and commuting mappings. Proc. Am. Math. Soc. 76(2), 333-338 (1979)

16. Jungck, G: Common fixed points for commuting and compatible maps on compacta. Proc. Am. Math. Soc. 103(3), 977-983 (1988)

17. Kubiak, T: Common fixed point theorems of pairwise commuting maps. Math. Nachr. 118, 123-127 (1984)

18. Boyd, DW, Wong, JS: On nonlinear contractions. Proc. Am. Math. Soc. 20, 458-464 (1969)

19. Meir, A, Keeler, E: A theorem on contraction mappings. J. Math. Anal. Appl. 28, 326-329 (1969)

20. Jachymski, J: Common fixed point theorems for some families of maps. Indian J. Pure Appl. Math. 25, 925-937 (1994)

21. Jachymski, J: Equivalent conditions and Meir-Keeler type theorems. J. Math. Anal. Appl. 194, 293-303 (1995)

22. Jachymski, J: Equivalence of some contractivity properties over metrical structures. Proc. Am. Math. Soc. 125, 2327-2335 (1997)

23. Jungck, G, Moon, KB, Park, S, Rhoades, BE: On generalizations of the Meir-Keeler type contraction maps: corrections. J. Math. Anal. Appl. 180, 221-222 (1993)

24. Pant, RP: A common fixed point theorem under a new condition. Indian J. Pure Appl. Math. 30(2), 147-152 (1999)

25. Pant, RP, Pant, V: A unified fixed point theorem. Bull. Calcutta Math. Soc. 91, 227-232 (1999)

26. Park, S, Rhoades, BE: Extension of some fixed point theorems of Hegedus and Kasahara. Math. Semin. Notes 9 , 113-118 (1981)

27. Park, S, Bae, JS: Extension of fixed point theorem of Meir and Keeler. Ark. Mat. 19, 223-228 (1981)

28. Pant, RP, Joshi, PC, Gupta, V: A Meir-Keeler type fixed point theorem. Indian J. Pure Appl. Math. 32(6), 779-787 (2001)

29. Jungck, G: Common fixed points for noncontinuous nonself maps on nonmetric spaces. Far East J. Math. Sci. 4, 199-215 (1996)

30. Jungck, G: Compatible mappings and common fixed points. Int. J. Math. Math. Sci. 9(4), 771-779 (1986)

31. Tivari, BML, Singh, SL: A note on recent generalizations of Jungck contraction principle. J. - Uttar Pradesh Gov. Coll. Acad. Soc. 3(1), 13-18 (1986) 
32. Pathak, HK: Weak* commuting mappings and fixed points. Indian J. Pure Appl. Math. 17(2), $201-211$ (1986)

33. Pathak, HK: Weak** commuting mappings and fixed points. J. Indian Acad. Math. 14(2), 94-98 (1992)

34. Jungck, G, Murthy, PP, Cho, YJ: Compatible mappings of type (A) and common fixed points. Math. Jpn. 38(2), 381-390 (1993)

35. Jungck, G, Pathak, HK: Fixed points via biased maps. Proc. Am. Math. Soc. 123(7), 2049-2060 (1995)

36. Pant, RP: Common fixed points of noncommuting mappings. J. Math. Anal. Appl. 188, 436-440 (1994)

37. Pathak, HK, Rai, D: Common fixed point theorems for PD-operator pairs under relaxed conditions with applications. J. Comput. Appl. Math. 239, 103-113 (2013)

38. Al-Thagafi, MA, Shahzad, N: Generalized /-nonexpansive selfmaps and invariant approximations. Acta Math. Sin. 24 867-876 (2008)

39. Hussain, N, Khamsi, MA, Latif, A: Common fixed points for $\mathrm{JH}$-operators and occasionally weakly biased pairs under relaxed conditions. Nonlinear Anal. 74, 2133-2140 (2011)

40. Pant, RP, Bisht, RK: Occasionally weakly compatible mappings and fixed points. Bull. Belg. Math. Soc. Simon Stevin 19, 655-661 (2012)

41. Bisht, RK, Shahzad, N: Faintly compatible mappings and common fixed points. Fixed Point Theory Appl. 2013, 156 (2013). doi:10.1186/1687-1812-2013-156

42. Sessa, S: On a weak commutativity condition of mappings in fixed point consideration. Publ. Inst. Math. (Belgr.) 32 149-153 (1982)

43. Pathak, HK: A Meir-Keeler type fixed point theorem for weakly uniformly contraction maps. Bull. Malays. Math. Soc. 13(1), 21-29 (1990)

44. Singh, SL, Mishra, SN: Coincidence points, hybrid fixed and stationary points of orbitally weakly dissipative maps. Math. Jpn. 39(3), 451-459 (1994)

45. Cho, YJ, Sharma, BK, Sahu, DR: Semi compatibility and fixed points. Math. Jpn. 42, 91-98 (1995)

46. Pathak, HK, Khan, MS: Compatible mappings of type $(B)$ and common fixed point theorems of Greguś type. Czechoslov. Math. J. 45(120)(4), 685-698 (1995)

47. Pathak, HK, Cho, YJ, Kang, SM, Lee, BS: Fixed point theorems for compatible mappings of type $(P)$ and applications to dynamic programming. Matematiche 50(1), 15-33 (1995)

48. Pathak, HK, Kang, SM, Cho, YJ, Jung, JS: Gregus type common fixed point theorems for compatible mappings of type $(T)$ and variational inequalities. Publ. Math. (Debr.) 46, 285-299 (1995)

49. Pathak, HK, Khan, M: A comparison of various types of compatible maps and common fixed points. Indian J. Pure Appl. Math. 28(4), 477-485 (1997)

50. Pathak, HK, Cho, YJ, Kang, SM: Remarks on R-weakly commuting mappings and common fixed point theorems. Bull. Korean Math. Soc. 34(2), 247-257 (1997)

51. Pathak, HK, Cho, YJ, Kang, SM: Common fixed points of biased maps of type $(A)$ and applications. Int. J. Math. Math. Sci. 21, 681-693 (1998)

52. Pathak, HK, Cho, YJ, Kang, SM, Madharia, B: Compatible mappings of type $(C)$ and common fixed point theorems of Greguś type. Demonstr. Math. 31(3), 499-518 (1998)

53. Fisher, B, Murthy, PP: Biased maps of type $\left(A_{f}\right)$ and fixed points. J. Nat. Sci. Math. 39, 33-42 (1999)

54. Dhage, BC: On common fixed point of coincidentally commuting mappings in D-metric space. Indian J. Pure Appl. Math. 30(4), 395-406 (1999)

55. Sastry, KPR, Krishna Murthy, ISR: Common fixed points of two partially commuting tangential selfmaps on a metric space. J. Math. Anal. Appl. 250(2), 731-734 (2000)

56. Shrivastava, PK, Bawa, NPS, Singh, P: Coincidence theorems for hybrid contraction II. Soochow J. Math. 26(4), 411-421 (2000)

57. Sahu, DR, Dhagat, VB, Srivastava, M: Fixed points with intimate mappings (I). Bull. Calcutta Math. Soc. 93(2), 107-114 (2001)

58. Singh, YR: Studies on fixed points, common fixed points and coincidences. PhD thesis, Manipur University, Kanchipur, India (2002)

59. Pathak, HK, Ume, JS: Weakly compatible mappings and common fixed point theorems with applications to variational inequalities. Adv. Nonlinear Var. Inequal. 10, 55-68 (2007)

60. Chen, J, Li, Z: Common fixed points for Banach operator pairs in best approximation. J. Math. Anal. Appl. 336 1466-1475 (2007)

61. Pant, V, Pant, RP: Common fixed points of conditionally commuting maps. Fixed Point Theory 11(1), 113-118 (2010)

62. Bouhadjera, H, Godet-Thobie, C: Common fixed point theorems for pair of subcompatible maps. arXiv:0906.3159v1 [math. FA] (2009)

63. Pathak, HK, Hussain, N: Common fixed points for $P$ operator pair with applications. Appl. Math. Comput. 217 3137-3143 (2010)

64. Suzuki, T, Pathak, HK: Almost biased mappings and almost compatible mappings are equivalent under some condition. J. Math. Anal. Appl. 368, 211-217 (2010)

65. Pathak, HK, Tiwari, R: A Gregus type common fixed point theorem in normed spaces with application. Banach J. Math. Anal. 5(1), 136-147 (2011)

66. Bouhadjera, H, Djoudi, A: Fixed point for occasionally weakly biased maps. Southeast Asian Bull. Math. 36, 489-500 (2012)

67. Pant, RP, Bisht, RK: Common fixed points of pseudo compatible mappings. Rev. R. Acad. Cienc. Exactas Fís. Nat., Ser. A Mat. (2013) doi:10.1007/s13398-013-0119-5

68. Haghi, RH, Rezapour, S, Shahzad, N: Some fixed point generalizations are not real generalizations. Nonlinear Anal. 74, 1799-1803 (2011)

69. Pant, RP: Discontinuity and fixed points. J. Math. Anal. Appl. 240, 284-289 (1999)

70. Aamri, M, El Moutawakil, D: Some new common fixed point theorems under strict contractive conditions. J. Math. Anal. Appl. 270, 181-188 (2002)

71. Sintunavarat, W, Kumam, P: Common fixed point theorems for a pair of weakly compatible mappings in fuzzy metric spaces. J. Appl. Math. 2011, Article ID 637958 (2011) 
72. Chauhan, S, Khan, MA, Kumar, S: Unified fixed point theorems in fuzzy metric spaces via common limit range property. J. Inequal. Appl. 2013, 182 (2013)

73. Chauhan, S, Radenović, S, Imdad, M, Vetro, C: Some integral type fixed point theorems in non-Archimedean Menger PM-spaces with common property (E.A) and applications of functional equations in dynamic programming. Rev. R. Acad. Cienc. Exactas Fís. Nat., Ser. A Mat. (2013). doi:10.1007/s13398-013-0142-6

74. Chauhan, S, Bhatnagar, S, Radenović, S: Common fixed point theorems for weakly compatible mappings in fuzzy metric spaces. Matematiche LXVIII(I), 87-98 (2013). doi:10.4418/2013.68.1.8

75. Imdad, M, Chauhan, S: Employing common limit range property to prove unified metrical fixed point theorems. Int. J. Anal. 2013, Article ID 763261 (2013)

76. Imdad, M, Chauhan, S, Kadelburg, Z: Fixed point theorems for mappings with common limit range property satisfying generalized $(\psi, \varphi)$-weak contractive conditions. Math. Sci. 7, 16 (2013). doi:10.1186/2251-7456-7-16

77. Chauhan, S, Kadelburg, Z, Dalal, S: A common fixed point theorem in metric space under general contractive condition. J. Appl. Math. 2013, Article ID 510691 (2013)

78. Sintunavarat, W, Kumam, P: Common fixed points for R-weakly commuting mappings in fuzzy metric spaces. Ann. Univ. Ferrara, Sez. 7: Sci. Mat. 58(2), 389-406 (2012)

79. Singh, SL: On common fixed points of commuting mappings. Math. Semin. Notes, Kobe Univ. 5(2), 131-134 (1977)

80. Bouhadjera, $\mathrm{H}$ : Common fixed point theorems for compatible mappings of type (C). Sarajevo J. Math. 1(14), 261-270 (2005)

81. Pathak, HK, Verma, RK: Weakly compatible mappings and Altman type contraction. Filomat 22(1), 33-46 (2008)

82. Pant, V, Bisht, RK: A new continuity condition and fixed point theorems with applications. Rev. R. Acad. Cienc. Exactas Fís. Nat., Ser. A Mat. (2013). doi:10.1007/s13398-013-0132-8

83. Pathak, HK, Cho, YJ, Chang, SS, Kang, SM: Compatible mappings of type $(P)$ and fixed point theorems in metric spaces and probabilistic metric spaces. Novi Sad J. Math. 26, 87-109 (1996)

84. Pathak, HK, Chang, SS, Cho, YJ: Fixed point theorems for compatible mappings of type (P). Indian J. Math. 36(2), 151-166 (1994)

85. Pathak, HK, Chang, SS, Cho, YJ: Fixed point theorems for mappings of type (P). Indian J. Math. 36, 151-166 (1994)

86. Pant, RP: Common fixed points of Lipschitz type mapping pairs. J. Math. Anal. Appl. 240, 280-283 (1999)

87. Chugh, R, Kumar, S: Common fixed points for weakly compatible maps. Proc. Indian Acad. Sci. Math. Sci. 111(2), 241-247 (2001)

88. Pathak, HK, Khan, MS, Tiwari, R: A common fixed point theorem and its application to nonlinear integral equations Comput. Math. Appl. 53, 961-971 (2007)

89. Murthy, PP: Important tools and possible applications of metric fixed point theory. Nonlinear Anal. 47, 3479-3490 (2001)

90. Jungck, G, Rhoades, BE: Fixed point theorems for occasionally weakly compatible mappings. Fixed Point Theory 7(2), 287-296 (2006) (Fixed Point Theory 9, 383-384 (2008) (erratum))

91. Bisht, RK, Pant, RP: A critical remark on 'Fixed point theorems for occasionally weakly compatible mappings'. J. Egypt. Math. Soc. 21, 273-275 (2013)

92. Dorić, D, Kadelburg, Z, Radenović, S: A note on occasionally weakly compatible and common fixed points. Fixed Point Theory 13, 475-480 (2012)

93. Abbas, M, Gopal, D, Radenović, S: A note on recently introduced commutative conditions. Indian J. Math. 55(2), 195-201 (2013)

94. Alghamdi, MA, Radenović, S, Shahzad, N: On some generalizations of commuting mappings. Abstr. Appl. Anal. 2012, Article ID 952052 (2012)

95. Bisht, RK, Rakocević, V: Some notes on PD-operator pairs. Math. Commun. 18, 441-445 (2013)

96. Kadelburg, Z, Radenović, S, Shahzad, N: A note on various classes of compatible-type pairs of mappings and common fixed point theorems. Abstr. Appl. Anal. 2013, Article ID 697151 (2013). doi:10.1155/2013/697151

97. Al-Thagafi, MA, Shahzad, N: A note on occasionally weakly compatible maps. Int. J. Math. Anal. 3(2), 55-58 (2009)

98. Abbas, M, Jungck, G: Common fixed point results for noncommuting mappings without continuity in cone metric spaces. J. Math. Anal. Appl. 341(1), 416-420 (2008)

99. Bielecki, A: Une remrque sur la methode de Banach-Cacciopoli-Tikhonov dans la theorie des euations differentielles ordinaries. Bull. Acad. Pol. Sci., Cl. III 4, 261-264 (1956)

100. Caratheodory, C: Vorlesungen über reele Funktionen. Teubner, Berlin (1918)

101. Meinardus, G: Invarianz bei linearen approximationen. Arch. Ration. Mech. Anal. 14, 301-303 (1963)

102. Schauder, J: Der Fixpunktsatz in Funktionalraumen. Stud. Math. 2, 171-180 (1930)

103. Brosowski, B: Fixpunktsatze in der Approximationstheorie. Mathematica 11, 195-220 (1969)

104. Subrahmanyam, PV: An application of a fixed point theorem to best approximation. J. Approx. Theory 20, 165-172 (1977)

105. Shahzad, N: A result on best approximation. Tamkang J. Math. 29, 223-226 (1998) corrections: Tamkang J. Math. 30 165 (1999)

106. Shahzad, N: Noncommuting maps and best approximations. Rad. Mat. 10, 77-83 (2001)

107. Shahzad, N: Invariant approximations and R-subweakly commuting maps. J. Math. Anal. Appl. 257(1), 39-45 (2001)

108. Al-Thagafi, MA, Shahzad, N: Noncommuting selfmaps and invariant approximations. Nonlinear Anal. 64(12), 2778-2786 (2006)

109. Singh, SP: Applications of fixed point theorems in approximation theory. In: Lakshmikantham, V (ed.) Applied Nonlinear Analysis, pp. 389-397. Academic Press, New York (1979)

110. Singh, SP: An application of a fixed point theorem to approximation theory. J. Approx. Theory 25, 89-90 (1979)

111. Hicks, TL, Humphries, MD: A note on fixed point theorems. J. Approx. Theory 34, 221-225 (1982)

112. Smoluk, A: Invariant approximations. Mat. Stosow. 17, 17-22 (1981) (in Polish)

113. Habiniak, L: Fixed point theorems and invariant approximations. J. Approx. Theory 56, $241-244$ (1989)

114. Shahzad, N, Bassindowa, G: Fixed point theorems for Suzuki-generalized nonexpansive mappings with applications. J. Nonlinear Convex Anal. 13(4), 657-666 (2012)

115. O'Regan, D, Shahzad, N: Invariant approximations for generalized /-contractions. Numer. Funct. Anal. Optim. 26(4-5), 565-575 (2005) 
116. Sahab, SA, Khan, MS, Sessa, S: A result in best approximation theory. J. Approx. Theory 55, 349-351 (1988)

117. Jungck, G, Sessa, S: Fixed point theorems in best approximation theory. Math. Jpn. 42, 249-252 (1995)

118. Al-Thagafi, MA: Common fixed points and best approximation. J. Approx. Theory 85, 318-323 (1996)

119. Beg, I, Abbas, M: Common fixed points and best approximation in convex metric spaces. Soochow J. Math. 33 729-738 (2007)

120. Chandok, S, Narang, TD: Some common fixed point theorems for Banach operator pairs with applications in best approximation. Nonlinear Anal. TMA 73, 105-109 (2010)

121. Narang, TD, Chandok, S: Common fixed points and invariant approximation of $R$-subweakly commuting maps in convex metric spaces. Ukr. Math. J. 62, 1585-1596 (1367-1376) (2010)

122. O'Regan, D, Shahzad, N: Coincidence points and invariant approximation results for multimaps. Acta Math. Sin. Engl. Ser. 23(9), 1601-1610 (2007)

123. Pathak, HK, Hussain, N: Common fixed points for Banach operator pairs with applications. Nonlinear Anal. 69 2788-2802 (2008)

124. Shahzad, N: Invariant approximations, generalized /-contractions, and R-subweakly commuting maps. Fixed Point Theory Appl. 1, 79-86 (2005)

125. Shahzad, N: On R-subweakly commuting maps and invariant approximations in Banach spaces. Georgian Math. J. 12, 157-162 (2005)

126. Shahzad, N, Markin, J: Invariant approximations for commuting mappings in CAT(0) and hyperconvex spaces. J. Math. Anal. Appl. 337(2), 1457-1464 (2008)

127. Singh, SP: Some results on best approximation in locally convex spaces. J. Approx. Theory 28, $72-76$ (1980)

128. Singh, SP, Watson, B, Srivastava, P: Fixed Point Theory and Best Approximation: The KKM-Map Principle. Kluwer Academic, Dordrecht (1997)

129. Pathak, HK, Khan, M, Liu, Z, Ume, JS: Fixed point theorems in metrically convex spaces and applications. J. Nonlinear Convex Anal. 4(2), 231-244 (2003)

130. Khan, A, Akbar, F: Common fixed points from best simultaneous approximations. Taiwan. J. Math. 13(5), 1379-1386 (2009)

131. Milman, PD: On best simultaneous approximation in normed linear spaces. J. Approx. Theory 20, $223-238$ (1977)

132. Nashine, HK: Applications of fixed point theorem to best simultaneous approximation in convex metric spaces. Kragujev. J. Math. 33, 107-118 (2010)

133. Sahney, BN, Singh, SP: On best simultaneous approximation. In: Approximation Theory III, pp. 783-789. Academic Press, San Diego (1980)

134. Sahney, BN, Singh, KL, Whitfield, JHM: Best approximations in locally convex spaces. J. Approx. Theory 38(2), 182-187 (1983)

135. Vijayaraju, P, Marudai, M: Some results on common fixed points and best approximations. Indian J. Math. 46, 233-244 (2004)

136. Vijayraju, P: Applications of fixed point theorem to best simultaneous approximations. Indian J. Pure Appl. Math. 24(1), 21-26 (1993)

137. Alghamdi, MA, Dhompongsa, S, Shahzad, N: Common fixed points for Suzuki-generalized nonexpansive maps. J. Appl. Math. 2013, Article ID 206759 (2013)

138. Beg, I, Shahzad, N, labal, M: Fixed point theorems and best approximation in convex metric space. Approx. Theory Its Appl. 8(4), 97-105 (1992)

139. Narang, TD, Chandok, S: Common fixed points and invariant approximations of pointwise $R$-subweakly commuting maps on nonconvex sets. Gen. Math. 18(4), 109-125 (2010)

140. Pathak, HK, Shahzad, N: Some results on best proximity points for cyclic mappings. Bull. Belg. Math. Soc. Simon Stevin 20(3), 559-572 (2013)

141. Pathak, HK: Applications of fixed point technique in solving certain dynamic programming and variational inequalities. Nonlinear Anal. (2005). doi:10.1016/j.na.2005.03.033

142. Daffer, PZ, Kaneko, H: Applications of $f$-contraction mappings to nonlinear integral equations. Bull. Inst. Math. Acad. Sin. 22, 69-74 (1994)

143. Pathak, HK, Mishra, SN, Kalinde, AK: Common fixed point theorems with applications to nonlinear integral equations. Demonstr. Math. XXXII(3), 547-564 (1999)

144. Pathak, HK: Integral $\phi$-type contractions and existence of continuous solutions for nonlinear integral inclusions. Nonlinear Anal. 71, 2577-2591 (2009)

10.1186/1687-1812-2014-38

Cite this article as: Agarwal et al.: A comparison of various noncommuting conditions in metric fixed point theory and their applications. Fixed Point Theory and Applications 2014, 2014:38 